\section{Application of Nonlinear Filtering to Navigation System Design Using Passive Sensors}

\section{ISAAC KAMINER}

WEI KANG, Member, IEEE

\section{OLEG YAKIMENKO}

Naval Postgraduate School

\section{ANTONIO PASCOAL}

Instituto Superior Tecnico

Portugal

\begin{abstract}
The problem of navigation system design for autonomous aircraft landing is addressed. New nonlinear filter structures are introduced to estimate the position and velocity of an aircraft with respect to a possibly moving landing site, such as a naval vessel, based on measurements provided by airborne vision and inertial sensors. By exploring the geometry of the navigation problem, the navigation filter dynamics are cast in the framework of linear parametrically varying systems (LPVs). Using this set-up, filter performance and stability are studied in an $H_{\infty}$ setting by resorting to the theory of linear matrix inequalities (LMIs). The design of nonlinear, regionally stable filters to meet adequate $H_{\infty}$ performance measures is thus converted into that of determining the feasibility of a related set of LMIs and finding a solution to them, if it exists. This is done by using widely available numerical tools that borrow from convex optimization techniques. The mathematical framework that is required for integrated vision/inertial navigation system design is developed and a design example for an air vehicle landing on an aircraft carrier is detailed.
\end{abstract}

Manuscript received September 13, 1999; revised June 1, 2000; released for publication July 22, 2000.

IEEE Log No. T-AES/37/1/02924.

Refereeing of this contribution was handled by P. K. Willett.

This work was supported by the Office of Naval Research under Contract N0001497AF00002. A. Pascoal was also supported by a NATO Fellowship during his stay at the Naval Postgraduate School.

Authors' addresses: I. Kaminer, Department of Aeronautical and Astronautical Engineering, Naval Postgraduate School, Monterey, CA 93943; W. Kang, Department of Mathematics, Naval Postgraduate School, Monterey, CA 93943; O. Yakimenko, Department of Aeronautical and Astronautical Engineering, Naval Postgraduate School, Monterey, CA 93943, currently on leave from Zhukovski Air Force Engineering Academy, Moscow, Russia; A. Pascoal, Department of Electrical Engineering and Institute for Systems and Robotics, Instituto Superior Tecnico, Av. Roviso Pais, 1096 Lisbon Cedex, Portugal.

U.S. Government work not protected by U.S. copyright.

0018-9251/01/\$10.00 2001 IEEE

\section{INTRODUCTION}

This work describes a solution to the problem of estimating the relative position and velocity of an autonomous aircraft with respect to a moving platform, such as a naval vessel, using passive sensors. The main motivation for this work stems from the need to develop reliable, miniaturized advanced navigation systems to enable the safe operation of unmanned air vehicles. Economy considerations, together with strict requirements imposed in the course of some envisioned mission scenarios, all but dictate the need to use passive sensors only, thus the emphasis on the integration of vision with other passive sensors such as altimeters and other inertial sensors installed on-board the aircraft.

For previous related work in this area, see [1-4] and the references therein. References [1,2] describe a solution to the problem of estimating the ground velocity and position of an aircraft based on visual terrain information, whereas $[3,4]$ focus on the use of GPS and vision based systems for aircraft navigation during night landing. Both papers tackle the problem of navigation system design in the context of extended Kalman filters.

An alternative approach to the navigation system design is proposed here. The problem is formulated in a deterministic setting and relies on the use of special nonlinear filter structures to estimate the relative position and velocity of an aircraft with respect to a moving landing site based on measurements provided by airborne vision and other inertial sensors. The key advantage of this approach is that the resulting nonlinear filters have guaranteed regional stability and performance. Furthermore, it has enabled us to establish a lower bound on the achievable $H_{\infty}$ filter performance and show that this bound is closely related to positional dilution of precision (PDOP). PDOP is commonly used in science of navigation to characterize the impact of the geometry on the accuracy of the navigation solution.

This work builds on a key result introduced in [5], where a useful property of the so-called perspective projection map is derived and used in the development of a visual estimation system for dexterous manipulation. By using that result and exploring the geometry of the navigation problem at hand, the nonlinear filter dynamics are cast in the framework of linear parametrically varying systems (LPVs) [6]. Using this set-up, filter performance and stability are studied in an $H_{\infty}$ setting by resorting to the theory of linear matrix inequalities (LMIs) [7]. The design of nonlinear, regionally stable filters to ensure stability and meet adequate $H_{\infty}$ performance measures is thus converted into that of determining the feasibility of a related set of LMIs and finding a solution to them, if it exists. This is done by using widely available numerical tools that borrow from convex optimization techniques. The mathematical 
framework is developed here that is required for integrated vision/inertial navigation system design in deterministic setting and a design example for an air vehicle on a final approach to an aircraft carrier is detailed.

The paper is organized as follows. Section II introduces some background material on the subject of LPV linear systems and their induced operator norms. Section III describes the class of integrated vision/inertial navigation systems that we consider and provides a rigorous mathematical formulation of the related filtering problems. Section IV provides solutions to the problems posed in terms of LMIs. Section $\mathrm{V}$ extends previous results to the case of stereo vision. Section VI includes an example illustrating application of the proposed techniques. Finally, Section VII contains the main conclusions and discusses theoretical and practical issues that deserve further consideration.

\section{MATHEMATICAL BACKGROUND}

This section summarizes the mathematical formalism that is required for the study of LPV systems, including their analysis in terms of induced operator norms. The notation is standard, see [8].

Let $\mathcal{G}: L_{2} \rightarrow L_{2}$ be a stable, linear time invariant (LTI) system with a minimal realization $\Sigma_{\mathcal{G}}:=$ $\{A, B, C\}$, and let $G(s)=C(s I-A)^{-1} B$ denote the corresponding transfer matrix. Then, the induced operator norm $\|\mathcal{G}\|_{(2, i)}$ equals the $\mathcal{H}_{\infty}$ norm of $G$, denoted $\|G\|_{\infty}$, where

$$
\|G\|_{\infty}:=\sup _{\omega \in \mathcal{R}} \sigma_{\max }\left(G^{T}(-j \omega) G(j \omega)\right)
$$

and $\sigma_{\max }(\cdot)$ denotes the maximum singular value of a matrix. Given a positive integer $\gamma>0$, then $\|\mathcal{G}\|_{(2, i)}<\gamma$ if and only if there exists a positive definite matrix $X$ that satisfies the matrix inequality [7]

$$
\left[\begin{array}{cc}
A^{T} X+X A+\frac{C^{T} C}{\gamma^{2}} & X B \\
B^{T} X & -I
\end{array}\right]<0 .
$$

The above inequality is an LMI in the matrix variable $X$. Checking for the existence of $X>0$ that satisfies (1) is easily done by resorting to widely available numerical algorithms [9]. Using Schur complements [7], (1) can be rewritten as

$$
A^{T} X+X A+X B B^{T} X+\frac{C^{T} C}{\gamma^{2}}<0 .
$$

We now extend the above concepts and tools to the set of LPV systems, that is, dynamical systems whose state-space entries depend continuously on a time-varying parameter. See $[6,7,10]$ and the references therein for an introduction to the subject. Let the parameter variation set $\mathcal{Q}$ be a compact subset of $\mathcal{R}^{p}$ and let $\mathcal{F}_{\rho}$ be the set of all continuous functions mapping $\mathcal{R}_{+}$to $\mathcal{Q}$. An LPV system $\mathcal{G}_{\mathcal{F}_{\rho}}$ admits a state space representation

$$
\mathcal{G}_{\mathcal{F}_{\rho}}=\left\{\begin{array}{l}
\dot{x}=A(\rho(t)) x+B(\rho(t)) w \\
z=C(\rho(t)) x
\end{array}\right.
$$

where $\rho \in \mathcal{F}_{\rho}, w$ is the input, $z$ is the ouptut, and $x$ is the state-space vector. For each $\rho(\cdot) \in \mathcal{F}_{\rho}$, (3) defines a linear time-varying system $\mathcal{G}_{\rho}$ with state transition matrix $\Phi_{\rho}(t, \tau)$. The concept of induced operator norms can now be easily extended to the LPV framework to obtain the results that follow. An input-output LPV system $\mathcal{G}_{\mathcal{F}_{\rho}}: L_{2} \rightarrow L_{2}$ is said to be stable if its $L_{2}$-induced operator norm

$$
\begin{aligned}
\left\|\mathcal{G}_{\mathcal{F}_{\rho}}\right\|_{(2, i)} & =\sup _{\rho \in \mathcal{Q}}\left\|\mathcal{G}_{\rho}\right\|_{(2, i)} \\
& =\sup _{\rho \in \mathcal{Q}} \sup \left\{\frac{\left\|\mathcal{G}_{\rho} f\right\|_{2}}{\|f\|_{2}}: f \in L_{2},\|f\|_{2} \neq 0\right\}
\end{aligned}
$$

is well defined and finite. In an analogous manner, a system $\mathcal{G}_{\mathcal{F}_{\rho}}: L_{2} \rightarrow L_{\infty}$ is said to be finite-gain stable if its $L_{(2, \infty)}$-induced operator norm

$$
\begin{aligned}
\left\|\mathcal{G}_{\mathcal{F}_{\rho}}\right\|_{(2, \infty), i} & =\sup _{\rho \in \mathcal{Q}}\left\|\mathcal{G}_{\rho}\right\|_{(2, \infty), i} \\
& =\sup _{\rho \in \mathcal{Q}} \sup \left\{\frac{\left\|\mathcal{G}_{\rho} f\right\|_{\infty}}{\|f\|_{2}}: f \in L_{2},\|f\|_{2} \neq 0\right\}
\end{aligned}
$$

is well defined and finite.

The computation of the $L_{2}$-induced norm of an LPV system is based on the following generalization of (2).

THEOREM 1 [6] Consider the LPV system $\mathcal{G}_{\mathcal{F}_{\rho}}$ of (3) and let $\gamma>0$. Suppose $\exists X=X^{T}>0$ such that for all $\rho \in \mathcal{Q}$

$$
\begin{gathered}
A^{T}(\rho(t)) X+X A(\rho(t))+X B(\rho(t)) B^{T}(\rho(t)) X \\
+\frac{C^{T}(\rho(t)) C(\rho(t))}{\gamma^{2}}<0 .
\end{gathered}
$$

Then

$$
\begin{aligned}
& \text { for } x(0)=0, w \in L_{2},\|w\|_{2}<1 \text { and for all } \rho \in \mathcal{Q} \\
& \qquad \lim _{t \rightarrow \infty} x(t)=0 \\
& \left\|\mathcal{G}_{\mathcal{F}_{\rho}}\right\|_{(2, i)}<\gamma .
\end{aligned}
$$

The computation of the induced $L_{(2, \infty)}$ norm of an LPV system (also referred to as generalized $H_{2}$ norm) is based on the following result.

THEOREM 2 [10] Consider the LPV system $\mathcal{G}_{\mathcal{F}_{\rho}}$ of (3) and let $\alpha>0$. Suppose that $\rho(t) \in \mathcal{Q}$ for all $t \geq 0$. Suppose $\exists Y=Y^{T}>0$ such that for all $\rho \in \mathcal{Q}$

$$
\begin{aligned}
& A^{T}(\rho(t)) Y+Y A(\rho(t))+Y B(\rho(t)) B^{T}(\rho(t)) Y<0 \\
& Y-\frac{C^{T}(\rho(t)) C(\rho(t))}{\alpha^{2}}>0 .
\end{aligned}
$$

Then $\left\|\mathcal{G}_{\mathcal{F}_{\rho}}\right\|_{(2, \infty), i}<\alpha$. 
We will also need the following result, which establishes an upper bound on the $L_{\infty}$ norm of the output signal of an LPV system given a non-zero initial condition.

THEOREM 3 Consider the LPV system $\mathcal{G}_{\mathcal{F}_{\rho}}$ of (3) with $w=0$, and let $\beta>0$. Suppose that $\rho(t) \in \mathcal{Q}$ for all $t \geq 0$. Suppose $\exists Z=Z^{T}>0$ such that for all $\rho \in \mathcal{Q}$

$$
\begin{aligned}
& A^{T}(\rho(t)) Z+Z A(\rho(t))<0 \\
& Z-\frac{C^{T}(\rho(t)) C(\rho(t))}{\beta^{2}}>0, \quad \text { and } \\
& x(0)^{T} Z x(0)<1 .
\end{aligned}
$$

Then $\|z\|_{\infty}<\beta$. Furthermore, $x(t) \rightarrow 0$ as $t \rightarrow \infty$.

PROOF The proof follows directly from the results reported in $[7,10]$.

REMARK 1 In this paper, the matrix $A$ depends on the state variables and the time $t$, i.e., $A=A(x, t)$. In this case, we define

$$
Q=\left\{x \mid x^{T} Z x<1\right\}
$$

for Theorem 3. Since $V(x)=x^{T} Z x$ is decreasing in $Q$ along a trajectory $x(t)$ of (3), we know that $x(t)$ never reaches the boundary of $Q$ if $x(0) \in Q$. Thus, the assumption $\rho \in Q$ in Theorem 3 can be removed. More specifically, if (6), (7), and (8) hold for $x \in Q$, then $x(t) \in Q$ and $\|z\|_{\infty}<\beta$ for all $t \geq 0$. Furthermore, $x(t) \rightarrow 0$ as $t \rightarrow \infty$.

REMARK 2 If $A=A(x, t)$ in Theorem 2, we define

$$
Q=\left\{x \mid x^{T} Y x<1\right\}, \quad V(x)=x^{T} Y x .
$$

Suppose that (4) holds in $Q$, then $x(0)=0$ implies that

$$
V(x(t))<\int_{0}^{t} w^{T} w d t \leq 1
$$

for all $t \geq 0$ if $x(t) \in Q$. Suppose that $V\left(x\left(t_{1}\right)\right)=1$ for some $t_{1}>0$, then $1<\int_{0}^{t_{1}} w^{T} w d t \leq 1$, which is a contradiction. Therefore, $x(t)$ never leaves the region $Q$ if (4) holds in $Q$. The condition $\rho \in \mathcal{Q}$ can be removed from Theorem 2. More specifically, if (4) and (5) hold in $Q$ for $x(0)=0$, then $x(t)$ stays in $Q$ and $\left\|\mathcal{G}_{\mathcal{F}_{\rho}}\right\|_{(2, \infty), i}<\alpha$.

\section{PROBLEM FORMULATION}

This section introduces the navigation problem that is the main focus of the paper and describes its mathematical formulation in terms of an equivalent filter design problem. For the sake of clarity, we first introduce some required notation and review the kinematic relationships of an aircraft/ship carrier ensemble, where the former is equipped with a visionbased system.

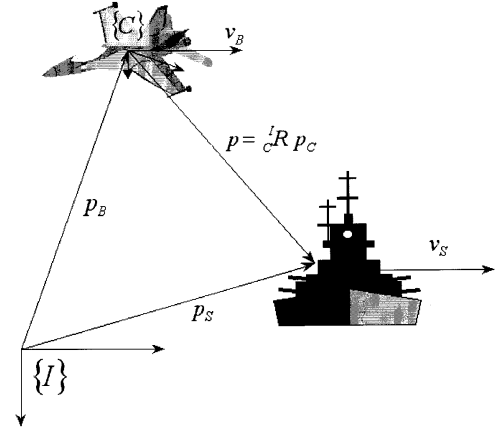

Fig. 1. Coordinate system.

\section{A. Notation}

Consider Fig. 1, which depicts an aircraft equipped with a vision camera operating in the vicinity of a ship. Let $\{\mathcal{I}\}$ denote an inertial reference frame, $\{\mathcal{B}\}$ a body-fixed frame that moves with the aircraft, and $\{\mathcal{C}\}$ a camera-fixed frame. The symbol $\{\mathcal{S}\}$ denotes a ship-fixed body frame. The following symbols are used.

$\mathbf{p}_{B}=\left[\begin{array}{lll}x_{b} & y_{b} & z_{b}\end{array}\right]^{T}$-position of the origin of $\{\mathcal{B}\}$ measured in $\{\mathcal{I}\}$ (i.e., inertial position of the aircraft).

$\mathbf{p}_{S}=\left[\begin{array}{lll}x_{s} & y_{s} & z_{s}\end{array}\right]^{T}$-inertial position of the ship.

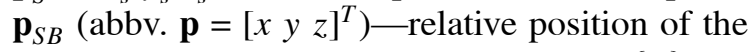
ship with respect to the aircraft, resolved in $\{\mathcal{I}\}$.

${ }^{C} \mathbf{p}_{S B}$ (abbv. $\left.\mathbf{p}_{C}=\left[\begin{array}{lll}x_{c} & y_{c} & z_{c}\end{array}\right]^{T}\right)$-relative position of the ship with respect to the aircraft, resolved in $\{\mathcal{C}\}$.

$\mathbf{v}_{B}$ - linear velocity of the origin of $\{\mathcal{B}\}$ measured in $\{\mathcal{I}\}$ (i.e., inertial velocity of the aircraft).

$\mathbf{v}_{S}$-inertial velocity of the ship.

${ }^{B} \mathbf{a}$-linear acceleration of $\{\mathcal{B}\}$ with respect to $\{\mathcal{I}\}$, resolved in $\{\mathcal{B}\}$.

$\omega$-angular velocity of $\{\mathcal{C}\}$ with respect to $\{\mathcal{I}\}$, resolved in $\{\mathcal{I}\}$.

$\lambda=\left[\begin{array}{lll}\phi & \theta & \psi\end{array}\right]^{T}$-vector of roll, pitch, and yaw angles that parameterize locally the orientation of frame $\{\mathcal{C}\}$ with respect to $\{\mathcal{I}\}$.

Given two frames $\{\mathcal{A}\}$ and $\{\mathcal{B}\},{ }_{B}^{A} R$ denotes the rotation matrix from $\{\mathcal{B}\}$ to $\{\mathcal{A}\}$. In particular, ${ }_{C}^{I} R$ (abbreviated $R$ ) is the rotation matrix from $\{\mathcal{C}\}$ to $\{\mathcal{I}\}$, parameterized locally by $\lambda$, that is, $R=R(\lambda)$.

\section{B. Kinematic Relations}

The rotation matrix $R$ satisfies the orthonormality condition $R^{T} R=I$. Furthermore, [11]:

$$
\dot{R}=R S(\omega)
$$

where

$$
S(\boldsymbol{\omega}):=\left[\begin{array}{ccc}
0 & -\omega_{z} & \omega_{y} \\
\omega_{z} & 0 & -\omega_{x} \\
-\omega_{y} & \omega_{x} & 0
\end{array}\right]
$$

is a skew symmetric matrix, that is, $S^{T}=-S$. The matrix $S$ satisfies the relationship $S(a) b=a \times b$, where 


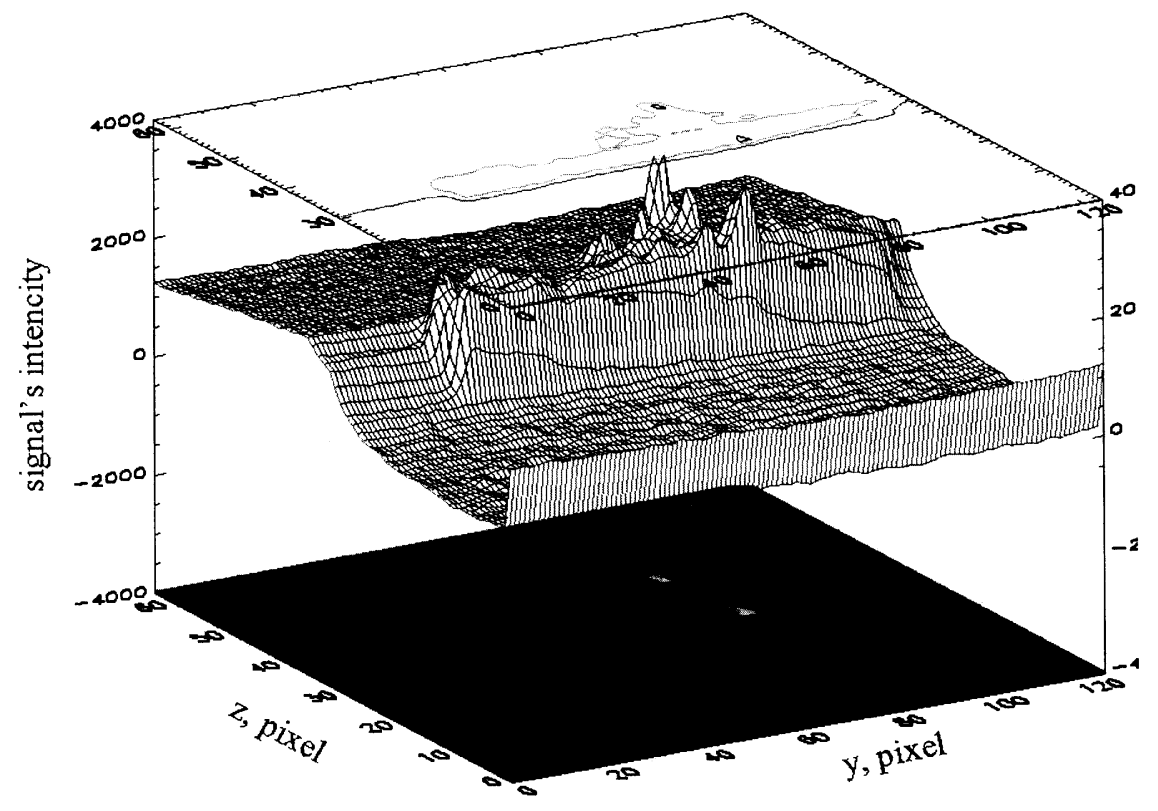

Fig. 2. IR image of ship.

$a, b$ are arbitrary vectors and $\times$ denotes the cross product operation. Furthermore, $\|S(\omega)\|=\|\omega\|$.

We introduce the following assumption.

A1. The ship's inertial velocity $\mathbf{v}_{S}$ is a non-zero constant.

From the above definitions, it follows that

$$
\mathbf{p}_{S}=\mathbf{p}_{B}+{ }_{C}^{I} R \mathbf{p}_{C} \Rightarrow \frac{d^{2}}{d t^{2}}{ }_{C}^{I} R \mathbf{p}_{C}=\frac{d^{2}}{d t^{2}} \mathbf{p}_{S}-\frac{d^{2}}{d t^{2}} \mathbf{p}_{B}
$$

and since $\left(d^{2} / d t^{2}\right) \mathbf{p}_{S}=0$ (assumption A1) we obtain

$$
\frac{d^{2}}{d t^{2}}\left({ }_{C}^{I} R \mathbf{p}_{C}\right)=-\frac{d^{2}}{d t^{2}} \mathbf{p}_{B} .
$$

Equation (12) shows that aside from a change in sign, the relative acceleration of the ship with respect to the aircraft resolved in $\{\mathcal{I}\}$ is equal to the aircraft's inertial acceleration resolved in $\{\mathcal{I}\}$. However, in the case of strapdown inertial navigation systems widely in use today [12] the aircraft's inertial acceleration is usually given in $\{\mathcal{B}\}$. Therefore, since

it follows that

$$
\frac{d^{2}}{d t^{2}} \mathbf{p}_{B}={ }_{B}^{I} R^{B} \mathbf{a}
$$

$$
\frac{d^{2}}{d t^{2}}\left({ }_{C}^{I} R \mathbf{p}_{C}\right)=-{ }_{B}^{I} R^{B} \mathbf{a}
$$

\section{Process Model}

The nonlinear filters developed here provide the relative position and velocity of an aircraft with respect to a point on the ship. This information, together with the aircraft's inertial velocity, is sufficient to estimate the ship's inertial velocity and, therefore, its heading. As argued in [13], in the unstructured environment of sea operations the best way to find a ship is by using an IR (infrared) camera. As is shown in Fig. 2, simple thresholding of an IR image will easily provide information on the coordinates of the centroid of the ship's hottest region (usually its smokestack or boiler room). Therefore, it is only natural that the origin of the ship's coordinate system $\mathcal{S}$ be attached to that point. It is with respect to this same point that the proposed nonlinear filters obtain relative position and velocity. In the immediate vicinity of the ship, where the relative orientation becomes critical, standard structure from motion solutions can be used [14].

We assume that the image of the origin of $\{\mathcal{S}\}$ acquired by a camera installed onboard the aircraft is obtained using a simple pinhole camera model of the form [15] (see Fig. 3)

$$
\left[\begin{array}{l}
u \\
v
\end{array}\right]=\pi_{f}\left(x_{c}, y_{c}, z_{c}\right)=\frac{f}{x_{c}}\left[\begin{array}{l}
y_{c} \\
z_{c}
\end{array}\right]
$$

where $f$ is the focal length of the camera and $\left[\begin{array}{ll}u & v\end{array}\right]^{T}$ are the image coordinates (the image plane coordinates of the smokestack, see Fig. 2) of $\mathbf{p}_{c}=$ $\left[\begin{array}{lll}x_{c} & y_{c} & z_{c}\end{array}\right]^{T}$ in the camera's image plane. We also make the following assumption.

A2. $\quad x_{c}>0$, that is, the ship is always located in front of the camera's image plane.

We further assume the following.

A3. The rotation matrices ${ }_{B}^{I} R$ and ${ }_{C}^{I} R$ are available from the onboard attitude measurement system.

This assumption is quite reasonable, considering the sophistication achieved by such systems today. 


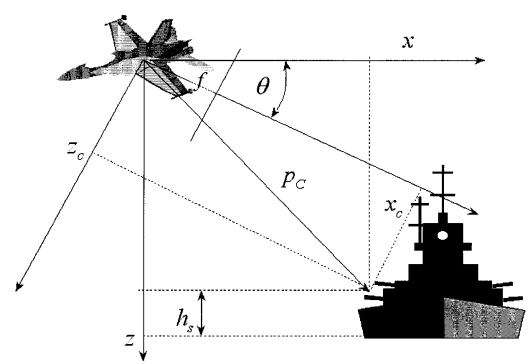

Fig. 3. Geometry of vision/altimeter process model for $\phi=0$.

Suppose the aircraft is equipped with a barometricbased sensor that provides a measurement of the altitude of the aircraft with respect to the mean sea level. Then, using the relation $\mathbf{p}={ }_{C}^{I} R \mathbf{p}_{c}$ and assuming that the aircraft is sufficiently away from the ship (so as to neglect the height $h_{s}$ of the ship's deck above the mean sea surface), we may assume the following.

$$
\text { A4. } h_{s}=0 \text {. }
$$

Thus we obtain that the altitude measurement equals

$$
z=g_{\phi, \theta}\left(\mathbf{p}_{c}\right)=\sin \theta x_{c}-\cos \theta \sin \phi y_{c}+\cos \theta \cos \phi z_{c}
$$

where $\phi$ and $\theta$ are the roll and pitch angles in the rotation matrix ${ }_{C}^{I} R$ (see Fig. 3).

We now introduce the underlying design model that plays a fundamental role in this work. Let $\mathbf{y}=$ $\left[\begin{array}{lll}u & v & z\end{array}\right]^{T}$. Then, the model that we consider can be written as

$$
\mathcal{G}=\left\{\begin{array}{l}
\frac{d}{d t} \mathbf{p}=\mathbf{v} \\
\frac{d}{d t} \mathbf{v}=-{ }_{B}^{I} R\left({ }^{B} \mathbf{a}_{m}+\mathbf{w}_{a}\right) \\
\mathbf{y}_{m}=g_{\phi, \theta}\left(\mathbf{p}_{C}\right)+\mathbf{w}_{y}
\end{array}\right.
$$

where $g_{\phi, \theta}: R^{3} \rightarrow R^{3}$ is defined by

$$
\left[\begin{array}{lll}
u & v & z
\end{array}\right]^{T}=g_{\phi, \theta}\left(\mathbf{p}_{C}\right)=\left[\begin{array}{ll}
\pi_{f}^{T} & g\left(\mathbf{p}_{c}\right)
\end{array}\right]^{T}
$$

and $\mathbf{a}_{m}$ and $\mathbf{y}_{m}$ denote the measured values of $\mathbf{a}$ and $\mathbf{y}$, respectively, the measurements being corrupted by the process noises $\mathbf{w}_{a}$ and $\mathbf{w}_{y}$. In what follows, the deterministic set-up of $H_{\infty}$ filtering [16] is adopted.

\section{Problem Definition}

The problem that we consider here consists of determining the relative position and relative velocity of an aircraft with respect to a landing site using vision and other onboard passive sensors. For the sake of clarity, we first tackle the simplified problem of designing a filter with no measurement noise in the model. This exercise is simple, yet it captures some of the key ideas used in the development that follows.

The additional notation that is required is introduced next. We let $\hat{\mathbf{p}}$ and $\hat{\mathbf{v}}$ denote estimates of $\mathbf{p}$ and $\mathbf{v}$, respectively. In the camera frame, they are denoted by $\hat{\mathbf{p}}_{C}, \hat{\mathbf{v}}_{C}$. We assume that the orientation of camera frame $\{\mathcal{C}\}$ with respect to $\{\mathcal{I}\}$ is restricted through the following set.

A5.

$$
\begin{gathered}
\Lambda_{C}=\left\{\boldsymbol{\lambda}=\left[\begin{array}{lll}
\phi & \theta & \psi
\end{array}\right]^{T}:|\phi| \leq \phi_{\max },\right. \\
\left.|\theta| \leq \theta_{\max },|\psi| \leq \psi_{\max }\right\} .
\end{gathered}
$$

Notice, for example, that $\psi_{\max }$ should be set to $\pi$. We further assume that the vectors $\mathbf{p}_{C}$ lie in the compact set as follows.

A6.

$$
\begin{gathered}
\mathcal{P}_{C}=\left\{\mathbf{p}_{C}=\left[\begin{array}{lll}
x_{C} & y_{C} & z_{C}
\end{array}\right], x_{\min } \leq x_{C} \leq x_{\max },\right. \\
\left.y_{\min } \leq y_{C} \leq y_{\max }, z_{\min } \leq z_{C} \leq z_{\max }\right\} .
\end{gathered}
$$

where $x_{\min }, \ldots z_{\max }$ are determined from the geometry of the problem at hand. The set $\mathcal{P}_{C}$ can be determined as follows. First, compute $\mathcal{P}_{C}$ for a nominal orientation of the camera (usually inertial orientation). Determine the maximum range of camera orientation angles with respect to the nominal orientation. Then compute $\mathcal{P}_{C}$ by allowing the angles to vary within these predetermined bounds.

Filter design will aim at ensuring that the estimates $\hat{\mathbf{p}}_{C}$ of $\mathbf{p}_{C}$ lie in a compact set

$$
\begin{aligned}
& \hat{\mathcal{P}}_{C}=\left\{\hat{\mathbf{p}}_{C}=\left[\begin{array}{lll}
\hat{x}_{C} & \hat{y}_{C} & \hat{z}_{C}
\end{array}\right],\right. \\
& \left|\hat{\mathbf{x}}_{C}-\mathbf{x}_{C}\right| \leq x_{\text {max }}-x_{\text {min }}+d x, \\
& \left|\hat{\mathbf{y}}_{C}-\mathbf{y}_{C}\right| \leq y_{\text {max }}-y_{\text {min }}+d y, \\
& \left.\left|\hat{\mathbf{z}}_{C}-\mathbf{z}_{C}\right| \leq z_{\max }-z_{\min }+d z\right\}
\end{aligned}
$$

where $d x, d y$ and $d z$ are positive numbers, and $d x<$ $x_{\min }$.

F1. Regional Stability: Consider the process model (15) and assume that $\mathbf{w}_{a}=\mathbf{w}_{y}=0$. For a given $\hat{\mathcal{P}}_{C}$, find a number $\alpha>0$, and a dynamical system (filter) $\mathcal{F}$ that operates on $\mathbf{y}_{m}$ and $\mathbf{a}_{m}$ to produce estimates $\hat{\mathbf{p}}$ of $\mathbf{p}$, and $\hat{\mathbf{v}}$ of $\mathbf{v}$, such that

$$
\begin{aligned}
& \hat{\mathbf{p}}_{C}(t) \in \hat{\mathcal{P}}_{C} \text { for any } t>0, \\
& \|\hat{\mathbf{p}}-\mathbf{p}\|+\|\hat{\mathbf{v}}-\mathbf{v}\| \rightarrow 0 \text { as } t \rightarrow \infty,
\end{aligned}
$$
provided that $\left\|\left(\hat{p}_{C}(0)-p_{C}(0), \hat{v}(0)-v(0)\right)^{T}\right\|<\alpha$.

Notice that the problem described aims at finding a filter that complements the information available from the vision system/barometric pressure sensor with that available from the inertial sensors.

The problem F1 focuses on the stability of the filter. The second filtering problem addresses the scenario where the performance of the filter in the presence of disturbances is considered.

F2. Regional Stability and Performance: Consider the process model (15) where $\mathbf{w}=\left[\begin{array}{ll}\mathbf{w}_{a} & \mathbf{w}_{y}\end{array}\right]^{T} \in L_{2}$, $\|\mathbf{w}\|_{2}<1$ and let the sets $\mathcal{P}_{C}$ and $\hat{\mathcal{P}}_{C}$ of allowable 
position vectors and allowable estimation vectors be given above. For given positive numbers $\gamma>0$ and $\alpha>0$, find a stable filter $\mathcal{F}$ that operates on $\mathbf{y}_{m}$ and $\mathbf{a}_{m}$ to obtain estimates $\hat{\mathbf{p}}$ of $\mathbf{p}$, and $\hat{\mathbf{v}}$ of $\mathbf{v}$ such that if $\left\|\left[(\hat{\mathbf{p}}(0)-\mathbf{p}(0))^{T} \quad(\hat{\mathbf{v}}(0)-\mathbf{v}(0))^{T}\right]^{T}\right\|<\alpha$, the filter satisfies the following conditions for all $\mathbf{w} \in \mathbf{L}_{2}$, $\|w\|_{2}<1$

$$
\begin{aligned}
& \hat{\mathbf{p}}_{C}(t) \in \hat{\mathcal{P}}_{C} \text { for all } t \geq 0, \\
& \text { If } \mathbf{w}=0, \text { then }\|\hat{\mathbf{p}}(t)-\mathbf{p}(t)\|+\|\hat{\mathbf{v}}(t)-\mathbf{v}(t)\| \rightarrow 0 \text { as }
\end{aligned}
$$
$t \rightarrow \infty$,

$\left\|T_{\mathbf{e w}}\right\|_{(2, i)}<\gamma$, where $\mathbf{e}:=\hat{\mathbf{p}}-\mathbf{p}$ is the estimation error and $T_{\text {ew }}: \mathbf{w} \rightarrow \mathbf{e}$.

Notice the technical requirement that an allowable set of position estimates $\hat{\mathcal{P}}_{C}$ be specified. As is shown later, this requirement is essential to establishing the boundedness of a certain operator for all possible values of the estimates $\hat{\mathbf{p}}(t)$. In practice, the "size" of the allowable region $\hat{\mathcal{P}}$ plays the role of a design parameter.

\section{PROPOSED SOLUTION}

This section describes the solutions to problems F1 and F2. First, however, we need the following basic results. Let $H$ denote the Jacobian of $g_{\phi, \theta}$ with respect to $\mathbf{p}_{C}$. From the definition of $g_{\phi, \theta}$, it follows that

$$
H\left(\mathbf{p}_{C}\right)=\left[\begin{array}{ccc}
-\frac{f y_{C}}{x_{C}^{2}} & \frac{f}{x_{C}} & 0 \\
-\frac{f z_{C}}{x_{C}^{2}} & 0 & \frac{f}{x_{C}} \\
-\sin \theta & -\cos \theta \sin \phi & \cos \theta \cos \phi
\end{array}\right] .
$$

It is easy to check that

$$
\operatorname{det}(H)=\frac{f^{2}}{x_{C}^{3}} z .
$$

Therefore, $H$ is not invertible if and only if $z=0$.

This implies that $H\left(\mathbf{p}_{C}\right)$ is invertible for all admissible values of $\mathbf{p}_{C}, \phi$ and $\theta$.

The next result is adopted from [5] and plays a key role in the development that follows. In particular, identity (19) makes it possible to show that the proposed nonlinear filter error dynamics represent an LPV system. This leads to the utilization of the LPV framework to reduce the estimation problem to that of determining the feasibility of a set of LMIs (see proofs of Theorems 4 and 5).

LEMMA 1 Let $g_{\phi, \theta}$ be given by (15). Then

$$
g_{\phi, \theta}\left(\hat{\mathbf{p}}_{C}\right)-g_{\phi, \theta}\left(\mathbf{p}_{C}\right)=L\left(\hat{\mathbf{p}}_{C}, \mathbf{p}_{C}\right) H\left(\hat{\mathbf{p}}_{C}\right)\left(\hat{\mathbf{p}}_{C}-\mathbf{p}_{C}\right)
$$

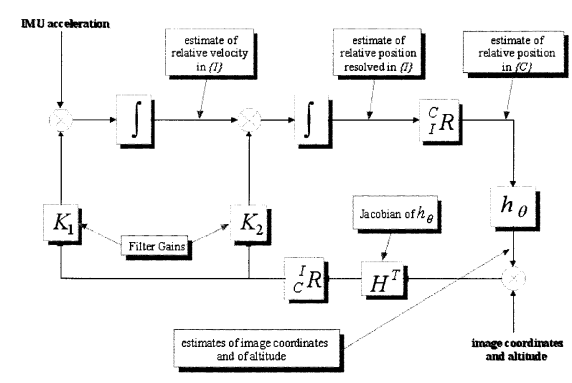

Fig. 4. Filtering structure: filters $\mathcal{F}_{1}$ and $\mathcal{F}_{2}$.

where $H$ is given in (18), $\hat{\mathbf{p}}_{C}=\left[\begin{array}{lll}\hat{x}_{c} & \hat{y}_{c} \hat{z}_{c}\end{array}\right]^{T}$ and

$$
L\left(\hat{\mathbf{p}}_{C}, \mathbf{p}_{C}\right)=\left[\begin{array}{ccc}
\frac{\hat{x}_{c}}{x_{c}} & 0 & 0 \\
0 & \frac{\hat{x}_{c}}{x_{c}} & 0 \\
0 & 0 & 1
\end{array}\right] .
$$

LEMMA 2 Let $\phi: R^{6} \rightarrow R^{3 \times 3}$ and $\phi_{1}: R^{3} \rightarrow R^{3 \times 3}$ be the operators defined by

$$
\phi\left(\hat{\mathbf{p}}_{C}, \mathbf{p}_{C}\right)=H^{T}\left(\hat{\mathbf{p}}_{C}\right) L\left(\hat{\mathbf{p}}_{C}, \mathbf{p}_{C}\right) H\left(\hat{\mathbf{p}}_{C}\right)
$$

and $\phi_{1}\left(\hat{\mathbf{p}}_{C}\right)=H^{T}\left(\hat{\mathbf{p}}_{C}\right) H\left(\hat{\mathbf{p}}_{C}\right)$. Then

$$
\begin{aligned}
\phi\left(\hat{\mathbf{p}}_{C}, \mathbf{p}_{C}\right)>0, \quad \phi_{1}\left(\hat{\mathbf{p}}_{C}\right)>0 \quad & \forall \quad \mathbf{p}_{C} \in \mathcal{P}_{C} \\
& \text { and } \hat{\mathbf{p}}_{C} \in \hat{\mathcal{P}}_{C} .
\end{aligned}
$$

PROOF The proof follows directly from assumptions A1-A 3 and the definitions of $H$ and $L$.

The following result provides a solution to problem F1.

THEOREM 4 Define a filter (see Fig. 4)

$$
\mathcal{F}_{1}=\left\{\begin{array}{l}
\dot{\hat{\mathbf{p}}}=\hat{\mathbf{v}}+K_{1 C}^{I} R H^{T}\left(\hat{\mathbf{p}}_{C}\right)\left(g_{\phi, \theta}\left(\hat{\mathbf{p}}_{C}\right)-\mathbf{y}_{m}\right) \\
\dot{\hat{\mathbf{v}}}=-{ }_{B}^{I} R^{B} \mathbf{a}_{m}+K_{2 C}^{I} R H^{T}\left(\hat{\mathbf{p}}_{C}\right)\left(g_{\phi, \theta}\left(\hat{\mathbf{p}}_{C}\right)-\mathbf{y}_{m}\right) \cdot \\
\hat{\mathbf{p}}_{C}={ }_{I}^{C} R \hat{\mathbf{p}}
\end{array}\right.
$$

Let $\hat{\mathcal{P}}_{C}$ be given. Let $\alpha<\min \left\{x_{\max }-x_{\min }+d x\right.$, $\left.y_{\max }-y_{\min }+d y, z_{\max }-z_{\min }+d z\right\}$ be a positive number. Define $r_{x}=\left(x_{\max }-x_{\min }+d x\right) / x_{\min }$, and $\epsilon=$ $\min _{\hat{\mathbf{p}}_{C} \in \hat{\mathcal{P}}_{C}} \lambda_{\min }\left(H^{T}\left(\hat{\mathbf{p}}_{C}\right) H\left(\hat{\mathbf{p}}_{C}\right)\right)$. Assume A1-A6 hold and $r_{x}<1$. Suppose there exists a matrix $P=P^{T} \in R^{6 \times 6}$ such that

$$
P>0
$$

$F^{T} P+P F+\left[\begin{array}{cc}-2\left(1-r_{x}\right)^{2} \epsilon & \\ 0 & 0\end{array}\right]<0$

$$
P-\max \left\{\frac{1}{\left(x_{\max }-x_{\min }+d x\right)^{2}}, \frac{1}{\left(y_{\max }-y_{\min }+d y\right)^{2}}\right. \text {, }
$$

$$
\left.\frac{1}{\left(z_{\max }-z_{\min }+d z\right)^{2}}\right\} C^{T} C>0
$$

$$
\frac{1}{\alpha^{2}}-P>0
$$


where

$$
F=\left[\begin{array}{ll}
0 & I \\
0 & 0
\end{array}\right]
$$

Let

$$
\left[\begin{array}{l}
K_{1} \\
K_{2}
\end{array}\right]=-P^{-1}\left(1-r_{x}\right) C^{T}, \quad C=\left[\begin{array}{l}
I \\
0
\end{array}\right] .
$$

Then the filter $\mathcal{F}_{1}$ solves the filtering problem $\mathrm{F} 1$.

ProOF Define the error state

$$
\begin{aligned}
& e_{1}=\hat{\mathbf{p}}-\mathbf{p} \\
& e_{2}=\hat{\mathbf{v}}-\mathbf{v} .
\end{aligned}
$$

Then, using Lemma 1 it follows that

$$
\begin{aligned}
\dot{e}_{1} & =e_{2}+K_{1 C}^{I} R H^{T}\left(\hat{\mathbf{p}}_{C}\right)\left(g_{\phi, \theta}\left(\hat{\mathbf{p}}_{C}\right)-g_{\phi, \theta}\left(\mathbf{p}_{C}\right)\right) \\
& =e_{2}+K_{1 C}^{I} R H^{T}\left(\hat{\mathbf{p}}_{C}\right) L\left(\hat{\mathbf{p}}_{C}, \mathbf{p}_{C}\right) H\left(\hat{\mathbf{p}}_{C}\right)\left(\hat{\mathbf{p}}_{C}-\mathbf{p}_{C}\right) \\
& =e_{2}+K_{1 C}^{I} R \phi\left(\hat{\mathbf{p}}_{C}, \mathbf{p}_{C}\right)_{C}^{I} R^{-1} e_{1}
\end{aligned}
$$

and

$$
\dot{e}_{2}=K_{2 C}^{I} R \phi\left(\hat{\mathbf{p}}_{C}, \mathbf{p}_{C}\right)_{C}^{I} R^{-1} e_{1} .
$$

The error dynamics can be written as

$$
\frac{d}{d t}\left[\begin{array}{l}
e_{1} \\
e_{2}
\end{array}\right]=\left(F+K_{C}^{I} R \phi\left(\hat{\mathbf{p}}_{C}, \mathbf{p}_{C}\right)_{C}^{I} R^{-1} C\right)\left[\begin{array}{l}
e_{1} \\
e_{2}
\end{array}\right]
$$

where $C=[I, 0]$ and $K=\left[K_{1}^{T}, K_{2}^{T}\right]^{T}$. Notice, the error dynamics given by (26) represent an LPV system that depends on $\left(\mathbf{p}_{C}, e\right)$. Now, to show that $\hat{\mathbf{p}}_{C} \in \hat{\mathcal{P}}_{C}$, it is sufficient to show that $\left\|e_{1}\right\|<\min \left(x_{\max }-x_{\min }+\right.$ $\left.d x, y_{\max }-y_{\min }+d y, z_{\max }-z_{\min }+d z\right)$. From Theorem 3 and Remark 1, $\left\|e_{1}\right\|$ is bounded by $\min \left(x_{\max }-x_{\min }+\right.$ $\left.d x, y_{\max }-y_{\text {min }}+d y, z_{\max }-z_{\min }+d z\right)$ if there exists a matrix $P>0$ such that the following inequalities

$$
\begin{aligned}
& \begin{aligned}
&(F+\left.K_{C}^{I} R \phi\left(\hat{\mathbf{p}}_{C}, \mathbf{p}_{C}\right)_{C}^{I} R^{-1} C\right)^{T} P \\
&+P\left(F+K_{C}^{I} R \phi\left(\hat{\mathbf{p}}_{C}, \mathbf{p}_{C}\right)_{C}^{I} R^{-1} C\right)<0 \\
& P-\max \left\{\frac{1}{\left(x_{\max }-x_{\min }+d x\right)^{2}}, \frac{1}{\left(y_{\max }-y_{\min }+d y\right)^{2}},\right. \\
&\left.\frac{1}{\left(z_{\max }-z_{\min }+d z\right)^{2}}\right\} C^{T} C>0
\end{aligned} \\
& e(0)^{T} P e(0)<1
\end{aligned}
$$

hold in the set $Q=\left\{e \mid e^{T} P e<1\right\}$. From the assumption $\|e(0)\|<\alpha$ and the condition (24), the third inequality holds. The second inequality is given by (23). In the following, we focus on the solvability of the first inequality in the set $Q$. From (25), the first inequality becomes

$$
\begin{aligned}
F^{T} P & +P F+\left[\begin{array}{cc}
-2\left(1-r_{x}\right)_{C}^{I} R H^{T}\left(\hat{\mathbf{p}}_{C}\right) L H\left(\hat{\mathbf{p}}_{C}\right)_{C}^{I} R^{-1} & 0 \\
0 & 0
\end{array}\right] \\
& <0 .
\end{aligned}
$$

In the set $Q$ (23) implies $\left\|e_{1}\right\|<\min \left(x_{\max }-x_{\min }+d x\right.$, $\left.y_{\text {max }}-y_{\text {min }}+d y, z_{\max }-z_{\text {min }}+d z\right)$. So

$$
L=I+\left[\begin{array}{ccc}
\frac{\hat{x}_{C}-x_{C}}{x_{C}} & 0 & 0 \\
0 & \frac{\hat{x}_{C}-x_{C}}{x_{C}} & 0 \\
0 & 0 & 0
\end{array}\right]>1-r_{x}
$$

in $Q$. Therefore,

$$
\begin{aligned}
& -2\left(1-r_{x}\right)_{C}^{I} R H^{T}\left(\hat{\mathbf{p}}_{C}\right) L H\left(\hat{\mathbf{p}}_{C}\right)_{C}^{I} R^{-1} \\
& <-2\left(1-r_{x}\right)_{C}^{2 I} R H^{T}\left(\hat{\mathbf{p}}_{C}\right) H\left(\hat{\mathbf{p}}_{C}\right)_{C}^{I} R^{-1} \\
& <-2\left(1-r_{x}\right)^{2} \epsilon I .
\end{aligned}
$$

So, (22) implies (28) in the set $Q$. Now, from Theorem 3 and Remark 1, it follows that $\left\|e_{1}\right\|<$ $\min \left(x_{\max }-x_{\min }+d x, y_{\max }-y_{\text {min }}+d y, z_{\max }-z_{\min }+\right.$ dz) $\forall t \geq 0$ and $e(t) \rightarrow 0$ as $t \rightarrow \infty$.

The solvability of the inequality (22) is addressed in Theorem 6. It shows that the inequality has a solution if and only if $r_{x}<1$. Notice that the filter $\mathcal{F}_{1}$ complements the information available from the vision system/barometric pressure sensor with that available from the inertial sensors. This structure is similar to that found in complementary filters [17, 18], the practical relevance of which can hardly be overemphasized.

The next theorem provides solution to the filtering problem F2.

THEOREM 5 Let $\hat{\mathcal{P}}_{C}$ be given. Let $r_{x}$ and $\alpha$ be positive numbers as in Theorem 4. Define

$$
\epsilon=\min _{\hat{\mathbf{p}}_{C} \in \hat{\mathcal{P}}_{C}} \lambda_{\min }\left\{\phi_{1}\left(\hat{\mathbf{p}}_{C}\right)\right\} .
$$

Suppose assumptions A1-A6 hold. For a given gain $\gamma$, suppose there exists a matrix $P=P^{T} \in R^{6 \times 6}$ such that

$$
\begin{aligned}
& P>0
\end{aligned}
$$

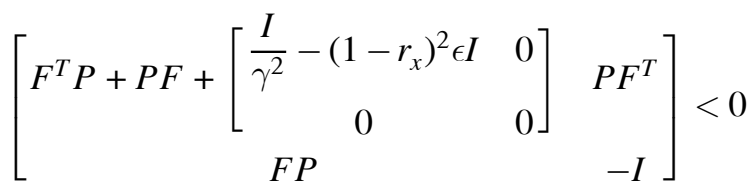

$$
\begin{aligned}
& P-4 \max \left\{\frac{1}{\left(x_{\max }-x_{\min }+d x\right)^{2}}, \frac{1}{\left(y_{\max }-y_{\min }+d y\right)^{2}},\right. \\
& \left.\frac{1}{\left(z_{\max }-z_{\min }+d z\right)^{2}}\right\} C^{T} C>0 \\
& \frac{1}{\alpha^{2}}-P>0
\end{aligned}
$$

Let

$$
\left[\begin{array}{l}
K_{1} \\
K_{2}
\end{array}\right]=-P^{-1}\left(1-r_{x}\right) C^{T}
$$


Define the filter (see Fig. 4)

$$
\mathcal{F}_{2}=\left\{\begin{array}{l}
\dot{\hat{\mathbf{p}}}=\hat{\mathbf{v}}+K_{1 C}^{I} R H^{T}\left(\hat{\mathbf{p}}_{C}\right)\left(g_{\phi, \theta}\left(\hat{\mathbf{p}}_{C}\right)-\mathbf{y}_{m}\right) \\
\dot{\hat{\mathbf{v}}}=-{ }_{B}^{I} R^{B} \mathbf{a}_{m}+K_{2 C}^{I} R H^{T}\left(\hat{\mathbf{p}}_{C}\right)\left(g_{\phi, \theta}\left(\hat{\mathbf{p}}_{C}\right)-\mathbf{y}_{m}\right) . \\
\hat{\mathbf{p}}_{C}={ }_{I}^{C} R \hat{\mathbf{p}}
\end{array}\right.
$$

Then $\mathcal{F}_{2}$ solves the filtering problem $\mathrm{F} 2$ if

$$
\left\|\left[(\hat{\mathbf{p}}(0)-\mathbf{p}(0))^{T} \quad(\hat{\mathbf{v}}(0)-\mathbf{v}(0))^{T}\right]^{T}\right\|<\alpha .
$$

PROOF Define the error states

$$
\begin{aligned}
& \mathbf{e}_{1}=\left[\begin{array}{lll}
e_{x} & e_{y} & e_{z}
\end{array}\right]^{T}=\hat{\mathbf{p}}-\mathbf{p} \\
& \mathbf{e}_{2}=\hat{\mathbf{v}}-\mathbf{v} .
\end{aligned}
$$

Using Lemma 1, simple algebraic manipulations show that

$$
\begin{aligned}
\dot{\mathbf{e}}_{1}= & \mathbf{e}_{2}+K_{1 C}^{I} R H^{T}\left(\hat{\mathbf{p}}_{C}\right)\left(g_{\phi, \theta}\left(\hat{\mathbf{p}}_{C}\right)-g_{\phi, \theta}\left(\mathbf{p}_{C}\right)-\mathbf{w}_{y}\right) \\
= & \mathbf{e}_{2}+K_{1 C}^{I} R H^{T}\left(\hat{\mathbf{p}}_{C}\right) L\left(\hat{\mathbf{p}}_{C}, \mathbf{p}_{C}\right) H\left(\hat{\mathbf{p}}_{C}\right)\left(\hat{\mathbf{p}}_{C}-\mathbf{p}_{C}\right) \\
& -K_{1 C}^{I} R H^{T}\left(\hat{\mathbf{p}}_{C}\right) \mathbf{w}_{y} \\
= & \mathbf{e}_{2}+K_{1 C}^{I} R \phi\left(\hat{\mathbf{p}}_{C}, \mathbf{p}_{C}\right)_{C}^{I} R^{-1} \mathbf{e}_{1}-K_{1 C}^{I} R H^{T}\left(\hat{\mathbf{p}}_{C}\right) \mathbf{w}_{y} .
\end{aligned}
$$

Similarly,

$$
\begin{aligned}
\dot{\mathbf{e}}_{2}= & { }_{B}^{I} R \mathbf{w}_{a}+K_{2 C}^{I} R \phi\left(\hat{\mathbf{p}}_{C}, \mathbf{p}_{C}\right)_{C}^{I} R^{-1} \mathbf{e}_{1} \\
& -K_{2 C}^{I} R H^{T}\left(\hat{\mathbf{p}}_{C}\right) \mathbf{w}_{y} .
\end{aligned}
$$

Then, the error dynamics admit the state-space realization

$$
\begin{aligned}
\frac{d}{d t}\left[\begin{array}{l}
\mathbf{e}_{1} \\
\mathbf{e}_{2}
\end{array}\right]= & \left(F+K_{C}^{I} R \phi\left(\hat{\mathbf{p}}_{C}, \mathbf{p}_{C}\right)_{C}^{I} R^{-1} C\right)\left[\begin{array}{l}
\mathbf{e}_{1} \\
\mathbf{e}_{2}
\end{array}\right] \\
& +\left[\left[\begin{array}{c}
0 \\
{ }_{B} R
\end{array}\right] \mid-K_{C}^{I} R H^{T}\left(\hat{\mathbf{p}}_{C}\right)\right] \mathbf{w} \\
\mathbf{e}= & C\left[\begin{array}{l}
\mathbf{e}_{1} \\
\mathbf{e}_{2}
\end{array}\right]
\end{aligned}
$$

where $\mathbf{w}=\left[\begin{array}{ll}\mathbf{w}_{a}^{T} & \mathbf{w}_{y}^{T}\end{array}\right]^{T}$ and $K=\left[\begin{array}{ll}K_{1}^{T} & K_{2}^{T}\end{array}\right]^{T}, C=\left[\begin{array}{ll}I & 0\end{array}\right]$. Notice, the error dynamics given by (37) represent an LPV system over the compact sets $\hat{\mathcal{P}}_{C} \times \mathcal{P}_{C} \times \Lambda_{C}$.

We now show that if the inequalities (32)-(35) are satisfied, then $\hat{\mathbf{p}}_{C}(t) \in \hat{\mathcal{P}}_{C}$ for all $t \geq 0, \mathbf{w} \in \mathbf{L}_{2}$, $\|\mathbf{w}\|_{2}<1$ provided $\left\|\left[(\hat{\mathbf{p}}(0)-\mathbf{p}(0))^{T}(\hat{\mathbf{v}}(0)-\mathbf{v}(0))^{T}\right]^{T}\right\|$ $<\alpha$. Since (37) is an LPV system, the variation of constants formula shows that its state-space response can be decomposed into two separate, additive terms where the first depends on the initial state $\mathbf{e}(0)$ only and the second term on the input function $\mathbf{w}$. We can thus use Theorems 2 and 3 to establish conditions under which there exists $\alpha>0$ and a matrix $K$ such that Claims 1 and 2 follow.

Claim 1: $\left\|e_{1}\right\|<\frac{1}{2} \min \left(x_{\max }-x_{\min }+d x, y_{\max }-\right.$ $\left.y_{\min }+d y, z_{\max }-z_{\min }+d z\right)$ in response to any $\|\mathbf{w}\|_{2}$ $<1$ and $\mathbf{e}(0)=0$.
Claim 2: $\quad\left\|e_{1}\right\|<\frac{1}{2} \min \left(x_{\max }-x_{\min }+d x, y_{\max }-\right.$ $\left.y_{\min }+d y, z_{\max }-z_{\min }+d z\right)$ in response to any $\left\|\left[(\hat{\mathbf{p}}(0)-\mathbf{p}(0))^{T}(\hat{\mathbf{v}}(0)-\mathbf{v}(0))^{T}\right]^{T}\right\|<\alpha$ and $\mathbf{w}=0$.

To prove Claim 1, we use Theorem 2 and Remark 2, in which $\left\|T_{e_{1} \mathbf{w}}\right\|_{i,(2, \infty)}<\frac{1}{2} \min \left(x_{\max }-x_{\min }+\right.$ $\left.d x, y_{\max }-y_{\text {min }}+d y, z_{\max }-z_{\min }+d z\right)$, if there exists $P=P^{T}>0$ such that

$$
\begin{aligned}
(F+ & \left.K_{C}^{I} R \phi\left(\hat{\mathbf{p}}_{C}, \mathbf{p}_{C}\right)_{C}^{I} R^{-1} C\right)^{T} P \\
& +P\left(F+K_{C}^{I} R \phi\left(\hat{\mathbf{p}}_{C}, \mathbf{p}_{C}\right)_{C}^{I} R^{-1} C\right) \\
& +P\left[\left[\begin{array}{c}
0 \\
{ }_{B} R
\end{array}\right] \mid-K_{C}^{I} R H^{T}(\hat{\mathbf{p}})\right] \\
& \times\left[\frac{\left[0_{B}^{I} R^{T}\right]}{\left(-K_{C}^{I} R H^{T}(\hat{\mathbf{p}})\right)^{T}}\right] P<0 \quad \text { and } \\
P- & 4 \frac{I}{\min \left(x_{\max }-x_{\min }+d x, y_{\max }-y_{\min }+d y, z_{\max }-z_{\min }+d z\right)} \\
& \times C^{T} C>0
\end{aligned}
$$

in $Q=\left\{e \mid e^{T} P e<1\right\}$. This is equivalent to

$$
\begin{aligned}
(F+ & \left.K_{C}^{I} R \phi\left(\hat{\mathbf{p}}_{C}, \mathbf{p}_{C}\right)_{C}^{I} R^{-1} C\right)^{T} P \\
& +P\left(F+K_{C}^{I} R \phi\left(\hat{\mathbf{p}}_{C}, \mathbf{p}_{C}\right)_{C}^{I} R^{-1} C\right) \\
& +P\left(F^{T} F+K_{C}^{I} R \phi_{1}\left(\hat{\mathbf{p}}_{C}\right)_{C}^{I} R^{-1} K^{T}\right) P<0, \quad \text { and } \\
P- & 4 \frac{I}{\min \left(x_{\max }-x_{\min }+d x, y_{\max }-y_{\min }+d y, z_{\max }-z_{\min }+d z\right)} \\
& \times C^{T} C>0
\end{aligned}
$$

in $Q$, where we used the relation

$$
\left[\begin{array}{c}
0 \\
I_{B} R
\end{array}\right]\left[\begin{array}{ll}
0 & { }_{B} R^{T}
\end{array}\right]=\left[\begin{array}{cc}
0 & 0 \\
0 & I
\end{array}\right]=F^{T} F .
$$

Inequality (39) follows the assumption (34). To simplify (38), we choose $K$ such that

$$
P K=-\left(1-r_{x}\right) C^{T}
$$

(this can always be done since $P>0$; the rationale for this choice is explained later). Then, the left-hand side of (38) is negative definite in $Q$ if

$$
\begin{aligned}
F^{T} P & +P F+\left[\begin{array}{cc}
-2\left(1-r_{x}\right)_{C}^{I} R \phi_{C}^{I} R^{-1}+\left(1-r_{x}\right)_{C}^{2 I} R \phi_{1 C}^{I} R^{-1} & 0 \\
0 & 0
\end{array}\right] \\
& +P F^{T} F P<0
\end{aligned}
$$

in $Q$. From (29) and (30), this inequality holds if

$$
\begin{aligned}
F^{T} P & +P F+P F^{T} F P \\
& +\left[\begin{array}{cc}
-\left(1-r_{x}\right)_{C}^{2 I} R H^{T}\left(\hat{\mathbf{p}}_{C}\right) H\left(\hat{\mathbf{p}}_{C}\right)_{C}^{I} R^{-1} & 0 \\
0 & 0
\end{array}\right]<0 .
\end{aligned}
$$


From Schur complements [7] and the definition (31), the inequality is true if

$$
\left[\begin{array}{cc|c}
F^{T} P+P F+\left[\begin{array}{cc}
-\left(1-r_{x}\right)^{2} \epsilon I & 0 \\
0 & 0
\end{array}\right] & P F^{T} \\
\hline F P & -I
\end{array}\right]<0 .
$$

From Theorem 2 and Remark 2, the feasibility of the LMIs (39), (42) implies $\left\|T_{e_{1} \mathbf{w}}\right\|_{i,(2, \infty)}<\frac{1}{2} \min \left(x_{\max }-\right.$ $\left.x_{\text {min }}+d x, y_{\text {max }}-y_{\text {min }}+d y, z_{\max }-z_{\text {min }}+d z\right)$. Since $\|\mathbf{w}\|_{2}<1$, we proved that

$$
\begin{gathered}
\left\|e_{1}\right\|<\frac{1}{2} \min \left(x_{\max }-x_{\min }+d x, y_{\max }-y_{\min }+d y,\right. \\
\left.z_{\max }-z_{\min }+d z\right)
\end{gathered}
$$

if the LMIs (32)-(34) hold and if

$$
\left[\mathbf{e}_{1}(0)^{T} \quad \mathbf{e}_{2}(0)^{T}\right]=0 .
$$

Claim 2 follows the proof of Theorem 4. Combining Claim 1 and 2, we proved that $\hat{\mathbf{p}}_{C}(t) \in$ $\hat{\mathcal{P}}_{C}$ for all $t \geq 0$ and all $\mathbf{w} \in \mathbf{L}_{2}$ if the inequalities (32)-(35) are satisfied and if $\|\left[(\hat{\mathbf{p}}(0)-\mathbf{p}(0))^{T}(\hat{\mathbf{v}}(0)-\right.$ $\left.\mathbf{v}(0))^{T}\right]^{T} \|<\alpha$.

Now, a similar application of Theorem 1 shows that if in addition the following LMI is feasible

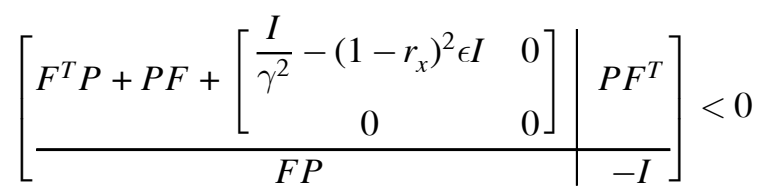

then $\left\|T_{\mathbf{z w}}\right\|_{i, 2}<\gamma$ and the error dynamics (37) are asymptotically stable for all $\left(\hat{\mathbf{p}}_{C}, \mathbf{p}_{C}\right) \in \hat{\mathcal{P}}_{C} \times \mathcal{P}_{C}$ and $\mathbf{w}=0$. Clearly, the feasibility of the LMI (43) guarantees that of (42).

REMARK 3 Consider expressions (38) and (42). Suppose $r_{x}=0$. Then

$$
\phi\left(\hat{\mathbf{p}}_{C}, \mathbf{p}_{C}\right)=\phi_{1}\left(\hat{\mathbf{p}}_{C}\right)
$$

and the inequality (38) can be rewritten as

$$
\begin{aligned}
F^{T} P & +\left(K R \phi_{1}\left(\hat{\mathbf{p}}_{C}\right) R^{-1} C\right)^{T} P+P F+P K R \phi_{1}\left(\hat{\mathbf{p}}_{C}\right) R^{-1} C \\
& +P F^{T} F P+P K R \phi_{1}\left(\hat{\mathbf{p}}_{C}\right) R^{-1} K^{T} P<0 .
\end{aligned}
$$

where $R={ }_{C}^{I} R$. Notice that

$$
\begin{aligned}
P R \phi_{1}\left(\hat{\mathbf{p}}_{C}\right) R^{-1} K^{T} P= & P K R \phi_{1}\left(\hat{\mathbf{p}}_{C}\right) R^{-1} C\left[\begin{array}{cc}
R \phi_{1}^{-1}\left(\hat{\mathbf{p}}_{C}\right) R^{-1} & 0 \\
0 & I
\end{array}\right] \\
& \times C^{T} R \phi_{1}\left(\hat{\mathbf{p}}_{C}\right) R^{-1} K^{T} P .
\end{aligned}
$$

Let

$$
W=P K R \phi_{1}\left(\hat{\mathbf{p}}_{C}\right) R^{-1} C .
$$

Then using completion of squares [8] it can be shown that inequality (44) is equivalent to

$$
\begin{aligned}
F^{T} P & +P F+P F^{T} F P+W+W^{T}+W\left[\begin{array}{cc}
\phi_{1}^{-1}\left(\hat{\mathbf{p}}_{C}\right) & 0 \\
0 & I
\end{array}\right] W^{T}<0 \\
\Longleftrightarrow & \\
F^{T} P & +P F+P F^{T} F P \\
& +\left(W+\left[\begin{array}{cc}
R \phi_{1}\left(\hat{\mathbf{p}}_{C}\right) R^{-1} & 0 \\
0 & I
\end{array}\right]\right)^{T}\left[\begin{array}{cc}
R \phi_{1}^{-1}\left(\hat{\mathbf{p}}_{C}\right) R^{-1} & 0 \\
0 & I
\end{array}\right] \\
& \times\left(W+\left[\begin{array}{cc}
R \phi_{1}\left(\hat{\mathbf{p}}_{C}\right) R^{-1} & 0 \\
0 & I
\end{array}\right]\right)-\left[\begin{array}{cc}
R \phi_{1}\left(\hat{\mathbf{p}}_{C}\right) R^{-1} & 0 \\
0 & I
\end{array}\right]<0 .
\end{aligned}
$$

The inequality (45) and definitions of $W$ and $C$ suggest that the best choice for $P K$ is

$$
P K=\left[\begin{array}{r}
-I \\
0
\end{array}\right]=-C^{T} .
$$

This observation motivated the choice of $P K$ in the proof of the Theorem 5 .

The next theorem derives necessary and sufficient conditions under which (33) is satisfied.

THEOREM 6 Let $F, \gamma$ and $\epsilon$ be defined in Theorem 5. Then $\exists P=P^{T}>0$ such that

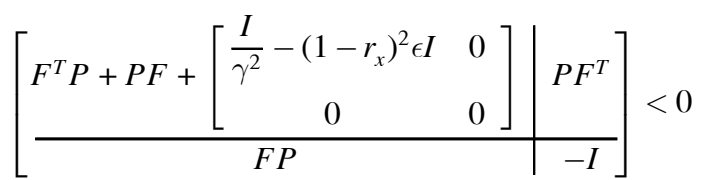

$$
\begin{aligned}
& \Longleftrightarrow \\
& \frac{I}{\gamma^{2}}-\left(1-r_{x}\right)^{2} \epsilon I<0 .
\end{aligned}
$$

Proof Suppose (46) holds for some $P>0$. Let

$$
P=\left[\begin{array}{ll}
P_{1} & P_{2} \\
P_{2}^{T} & P_{3}
\end{array}\right]
$$

and define $\delta=-\left(I / \gamma^{2}-\left(1-r_{x}\right)^{2} \epsilon I\right)$. Using simple algebra and Schur complements [7] it can be shown that (46) is negative definite iff

$$
F^{T} P+P F+\left[\begin{array}{cc}
-\delta I & 0 \\
0 & 0
\end{array}\right]+P F^{T} F P<0
$$

which is equivalent to

$$
\left[\begin{array}{cc}
\delta-P_{2} P_{2}^{T} & -P_{1}-P_{2} P_{3} \\
\left(-P_{1}-P_{2} P_{3}\right)^{T} & -P_{2}-P_{2}^{T}-P_{3}^{2}
\end{array}\right]>0
$$

Expression (48) clearly indicates that $\delta$ must be positive. To prove the converse assume that $\delta>0$ and consider two separate cases: $\delta \geq 1$ and $\delta<1$. 
Let $\delta<1$ and define

$$
P_{1}=\left[\begin{array}{cc}
\frac{2 \delta}{3} I & -\frac{\delta}{3} I \\
-\frac{\delta}{3} I & \frac{2 \delta}{3} I
\end{array}\right] .
$$

Then $P_{1}>0$. Now, by substituting (49) into (48) we obtain

$$
\begin{aligned}
& {\left[\begin{array}{ll}
\delta I-\frac{\delta^{2}}{9} I & \frac{2 \delta}{3} I-\frac{2 \delta^{2}}{9} I \\
\frac{2 \delta}{3} I-\frac{2 \delta^{2}}{9} I & \frac{2 \delta}{3} I-\frac{4 \delta^{2}}{9} I
\end{array}\right]>0} \\
& \Longleftrightarrow \\
& \delta-\frac{\delta^{2}}{9}>0 \\
& \frac{2 \delta}{3}-\frac{4 \delta^{2}}{9}>0 \\
& \frac{2}{9}-\frac{2 \delta}{9}>0
\end{aligned}
$$

and the result follows. For $\delta \geq 1$ it is easy to see that the choice

$$
P=\left[\begin{array}{cc}
\frac{2}{3} I & -\frac{1}{3} I \\
-\frac{1}{3} I & \frac{2}{3} I
\end{array}\right]
$$

leads to an identical result.

REMARK 4 Notice, that a similar argument as used in Theorem 6 can be used to show that the LMI

$$
F^{T} P+P F-\left[\begin{array}{cc}
\left(1-r_{x}\right)^{2} \epsilon I & 0 \\
0 & 0
\end{array}\right]
$$

used in Theorem 4 has a solution $P>0 \Longleftrightarrow r_{x}<1$.

REMARK 5 Theorem 6 shows that the LMI (46) is feasible iff

$$
\begin{aligned}
& \frac{I}{\gamma^{2}}-\left(1-r_{x}\right)^{2} \epsilon I<0 \\
& \Longleftrightarrow \\
& \gamma^{2}>\frac{1}{\left(1-r_{x}\right)^{2} \epsilon} .
\end{aligned}
$$

Recall that

$$
\begin{aligned}
\epsilon & =\min _{\hat{\mathbf{p}}_{C} \in \hat{\mathcal{P}}_{C}}\left\{\lambda_{\min }\left(H^{T}\left(\hat{\mathbf{p}}_{C}\right) H\left(\hat{\mathbf{p}}_{C}\right)\right)\right\} \\
& \leq \min _{\mathbf{p}_{C} \in \mathcal{P}_{C}}\left\{\lambda_{\min }\left(H^{T}\left(\mathbf{p}_{C}\right) H\left(\mathbf{p}_{C}\right)\right)\right\} \\
& \left.=\max _{\mathbf{p}_{C} \in \mathcal{P}_{C}}\left\{\left\|\left(H^{T}\left(\mathbf{p}_{C}\right) H\left(\mathbf{p}_{C}\right)\right)^{-1}\right\|\right)\right\}^{-1} .
\end{aligned}
$$

Therefore, we obtain

$$
\gamma^{2}>\frac{1}{\left(1-r_{x}\right)^{2}} \max _{\mathbf{p}_{C} \in \mathcal{P}_{C}}\left\{\left\|\left(H^{T}\left(\mathbf{p}_{C}\right) H\left(\mathbf{p}_{C}\right)\right)^{-1}\right\|\right\} .
$$

This inequality imposes a lower bound on the achievable values of $\gamma$. The bound is similar to the

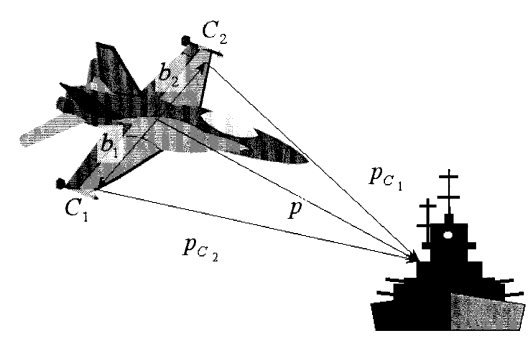

Fig. 5. Geometry of stereo vision process model.

classical PDOP metric that is commonly used in navigation systems to determine a lower bound on the achievable error covariance as a function of geometry of the underlying navigation problem $[12,19,20]$. Using our notation, the classical PDOP can be written as

$$
\text { PDOP }=\sqrt{\operatorname{tr}\left(H^{T}\left(\mathbf{p}_{C}\right) H\left(\mathbf{p}_{C}\right)\right)^{-1}} .
$$

We therefore see that the new bound derived in this work captures a worst case performance scenario and the estimate of $x_{c}$ increases the lower bound on the achievable $\gamma$, since

$$
1>\left(1-r_{x}\right)^{2}>0 .
$$

\section{INTEGRATION OF STEREO VISION WITH INERTIAL SENSORS}

When in the vicinity of the ship, using the barometric altimeter $g_{\phi, \theta}$ to determine the aircraft's altitude above the landing site may not be sufficient (i.e., assumption A4 must be lifted). In this case, the idea is to replace the altimeter with another camera and use stereo vision together with the accelerometers to estimate the aircraft's position with respect to the landing site.

Let $\mathcal{G}$ denote the process model for this problem and suppose that $\left\{\mathcal{C}_{1}\right\}$ and $\left\{\mathcal{C}_{2}\right\}$ denote coordinate systems attached to cameras 1 and 2, respectively (see Fig. 5). Then

$$
\mathcal{G}=\left\{\begin{aligned}
\frac{d}{d t} \mathbf{p} & =\mathbf{v} \\
\frac{d}{d t} \mathbf{v} & =-{ }_{B}^{I} R\left({ }^{B} \mathbf{a}_{m}+\mathbf{w}_{a}\right) \\
\mathbf{y}_{m} & =\left[\begin{array}{c}
\pi_{f}^{1}\left({ }_{I}^{C_{1}} R \mathbf{p}-\mathbf{b}_{1}\right) \\
\pi_{f}^{2}\left({ }_{I}^{C_{2}} R \mathbf{p}-\mathbf{b}_{2}\right)
\end{array}\right]+\mathbf{w}_{y}
\end{aligned}\right.
$$

where $\pi_{f}^{1}$ and $\pi_{f}^{2}$ define projections of the origin of the ship coordinate system $\mathcal{S}$ onto the image plane of each camera. Notice, in the case of stereo vision it is more convenient to define the vector $\mathbf{p}$ (relative position of the ship with respect to the aircraft resolved in $\mathcal{I}$ ) to be attached to an arbitrary point on the aircraft, rather than to the origin of one of the camera frames as was done before. Hence, the vectors $\mathbf{b}_{1}$ and $\mathbf{b}_{2}$ define relative position of the origin of each camera frame with respect to the origin of $\mathbf{p}$. 
Then

$$
H(\mathbf{p})=\frac{d h}{d \mathbf{p}}=\left[\begin{array}{ll}
\frac{d \pi_{f}^{1}}{d \mathbf{p}_{C_{1}}}{ }_{I}{ }_{1} R \\
d \pi_{f}^{2} \\
\frac{{ }^{2}}{d \mathbf{p}_{C_{2}}}{ }_{I} R
\end{array}\right] \quad \text { and } \quad \phi_{1}(\hat{\mathbf{p}})=H^{T}(\mathbf{p}) H(\mathbf{p}) .
$$

In this section, we use the following notation

$$
H_{i}\left(\mathbf{p}_{C_{i}}\right)=\frac{d \pi_{f}^{i}}{d \mathbf{p}_{C_{i}}}, \quad R_{i}={ }_{C_{i}}^{I} R \quad \text { where } \quad i=1,2 .
$$

THEOREM 7 If the the point $\mathbf{p}$ is not on the line through the two cameras, the matrix $H$ (p) has full rank, which is three.

PROOF It is enough to prove that

$$
H x=0, \quad x \in R^{3}
$$

implies $x=0$. It is obvious that $H x=0$ implies $H_{1} R_{1}^{T} x=0$ and $H_{2} R_{2}^{T} x=0$. Define $y=R_{1}^{T} x$. Since $H_{1} y=0$, we have

$$
y=\lambda_{1}\left[\begin{array}{lll}
x_{C_{1}} & y_{C_{1}} & z_{C_{1}}
\end{array}\right]^{T}
$$

for some scalar $\lambda_{1}$. Therefore,

$$
x=R_{1} y=\lambda_{1} R_{1}\left[\begin{array}{l}
x_{C_{1}} \\
y_{C_{1}} \\
z_{C_{1}}
\end{array}\right]=\lambda_{1}\left(\mathbf{p}-R_{1} \mathbf{b}_{1}\right) .
$$

Similarly, $H_{2} R_{2}^{T} x=0$ implies $x=\lambda_{2}\left(\mathbf{p}-R_{2} \mathbf{b}_{2}\right)$ for some scalar $\lambda_{2}$. Therefore,

$$
\left(\lambda_{1}-\lambda_{2}\right) \mathbf{p}-\lambda_{1} R_{1} \mathbf{b}_{1}+\lambda_{2} R_{2} \mathbf{b}_{2}=0 .
$$

If $\lambda_{1}=\lambda_{2} \neq 0$, then $R_{1} \mathbf{b}_{1}=R_{2} \mathbf{b}_{2}$. This is to say that the two cameras are located at the same point, which is not true. Therefore, $\lambda_{1}-\lambda_{2} \neq 0$ or $\lambda_{1}=\lambda_{2}=0$. If $\lambda_{1}-\lambda_{2} \neq 0$, then

$$
\mathbf{p}=\frac{\lambda_{1}}{\lambda_{1}-\lambda_{2}} R_{1} \mathbf{b}_{1}-\frac{\lambda_{2}}{\lambda_{1}-\lambda_{2}} R_{2} \mathbf{b}_{2}
$$

Notice that

$$
\frac{\lambda_{1}}{\lambda_{1}-\lambda_{2}}-\frac{\lambda_{2}}{\lambda_{1}-\lambda_{2}}=1,
$$

this implies that the point $\mathbf{p}$ is located on the line through the centers of the two cameras. This is not true. Therefore, the only possibility left is $\lambda_{1}=\lambda_{2}=0$. This implies that $x=0$.

In the sequel we employ the definitions of the sets $\mathcal{P}_{C_{1}}$ and $\mathcal{P}_{C_{1}}$ proposed in Section IIID. Since two cameras are involved in the process, the relative orientation of the two cameras plays an important role. The relative orientation (of camera 2 with respect to camera 1 ) is defined by the matrix $R_{2}^{T} R_{1}$. Assume that the relative orientation is restricted by a compact set $\mathcal{O}$ in $S O$ (3) containing the identity matrix. The sets $\mathcal{P}_{C_{2}}$ and $\hat{\mathcal{P}}_{C_{2}}$ are defined by

$$
\mathcal{P}_{C_{2}}=\left\{R_{2}^{T} R_{1}\left(\mathbf{p}_{C_{1}}+\mathbf{b}_{1}\right)-\mathbf{b}_{2}: R_{2}^{T} R_{1} \in \mathcal{O}, \mathbf{p}_{C_{1}} \in \mathcal{P}_{C_{1}}\right\}
$$

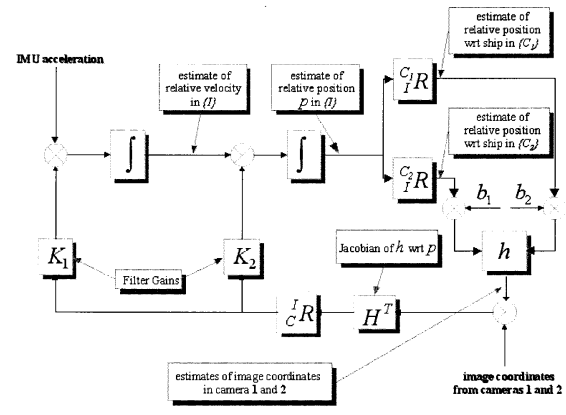

Fig. 6. Filtering structure: filter $\mathcal{F}_{3}$.

$$
\hat{\mathcal{P}}_{C_{2}}=\left\{R_{2}^{T} R_{1}\left(\hat{\mathbf{p}}_{C_{1}}+\mathbf{b}_{1}\right)-\mathbf{b}_{2}: R_{2}^{T} R_{1} \in \mathcal{O}, \hat{\mathbf{p}}_{C_{1}} \in \hat{\mathcal{P}}_{C_{1}}\right\} .
$$

For $\hat{\mathcal{P}}_{C_{2}}$, we assume $\left|\hat{x}_{C_{2}}-x_{C_{2}}\right|<\min \left\{x_{C_{2}}: \hat{\mathbf{p}}_{C_{2}} \in \hat{\mathcal{P}}_{C_{2}}\right\}$. Define

$$
r_{x}=\max _{\hat{\mathbf{p}}_{C_{2}} \in \hat{\mathcal{P}}_{C_{2}}, \mathbf{p}_{C_{2}} \in \mathcal{P}_{C_{2}}}\left\{\frac{x_{\max }-x_{\min }+d x}{x_{\min }}, \frac{\left|\hat{x}_{C_{2}}-x_{C_{2}}\right|}{x_{C_{2}}}\right\} .
$$

THEOREM 8 Given $\mathcal{P}_{C_{1}}, \hat{\mathcal{P}}_{C_{1}}$ and $\mathcal{O}$, let $r_{x}$ be the number defined above. Let $\alpha$ be a positive number such that $\alpha<\min \left(x_{\max }-x_{\min }+d x, y_{\max }-y_{\min }+d y, z_{\max }-\right.$ $\left.z_{\min }+d z\right)$. Define

$$
\begin{aligned}
\epsilon=\min _{\hat{\mathbf{p}}_{C_{1}} \in \hat{\mathcal{P}}_{C_{1}}, R_{2}^{T} R_{1} \in \mathcal{O}} \lambda_{\min }\left\{R_{2}^{T} R_{1} H_{1}^{T}\left(\hat{\mathbf{p}}_{C_{1}}\right) H_{1}\left(\hat{\mathbf{p}}_{C_{1}}\right) R_{1}^{T} R_{2}\right. \\
\left.+H_{2}^{T}\left(\hat{\mathbf{p}}_{C_{2}}\right) H_{2}\left(\hat{\mathbf{p}}_{C_{2}}\right)\right\} .
\end{aligned}
$$

Suppose assumptions A1-A3, A5-A6 hold and $r_{x}<1$.

For a given gain $\gamma$, suppose there exists a matrix

$P=P^{T} \in R^{6 \times 6}$ such that

$$
\begin{aligned}
& P>0 \\
& {\left[\begin{array}{cc|c}
F^{T} P+P F+\left[\begin{array}{cc}
\frac{I}{\gamma^{2}}-\left(1-r_{x}\right)^{2} \epsilon I & 0 \\
0 & 0
\end{array}\right] & P F^{T} \\
\hline F P & -I
\end{array}\right]<0} \\
& P-\frac{4}{\min \left(x_{\max }-x_{\min }+d x, y_{\text {max }}-y_{\text {min }}+d y, z_{\max }-z_{\text {min }}+d z\right)} \\
& \times C^{T} C>0 \text {, } \\
& \frac{1}{\alpha^{2}}-P>0 \text {. }
\end{aligned}
$$

Let

$$
\left[\begin{array}{l}
K_{1} \\
K_{2}
\end{array}\right]=-P^{-1}\left[\begin{array}{c}
\left(1-r_{x}\right) I \\
0
\end{array}\right] .
$$

The filter is defined by (see Fig. 6)

$$
\mathcal{F}_{3}=\left\{\begin{array}{l}
\dot{\hat{\mathbf{p}}}=\hat{\mathbf{v}}+K_{1} H^{T}\left(\hat{\mathbf{p}}_{C_{1}}, \hat{\mathbf{p}}_{C_{2}}\right)\left(h(\hat{\mathbf{p}})-\mathbf{y}_{m}\right) \\
\dot{\hat{\mathbf{v}}}=-{ }_{B}^{I} R^{B} \mathbf{a}_{m}+K_{2} H^{T}\left(\hat{\mathbf{p}}_{C_{1}}, \hat{\mathbf{p}}_{C_{2}}\right)\left(h(\hat{\mathbf{p}})-\mathbf{y}_{m}\right) \\
\hat{\mathbf{p}}_{C_{i}}=R_{i}^{T} \hat{\mathbf{p}}-\mathbf{b}_{i}, \quad i=1,2 .
\end{array}\right.
$$

Then $\mathcal{F}_{3}$ solves the filtering problem $\mathrm{F} 2$ using stereo vision and acceleration measurements if

$$
\left\|\left[(\hat{\mathbf{p}}(0)-\mathbf{p}(0))^{T} \quad(\hat{\mathbf{v}}(0)-\mathbf{v}(0))^{T}\right]^{T}\right\|<\alpha .
$$


Proof of Theorem 8 is similar to the proof of Theorem 5 and is omitted for the sake of brevity.

REMARK 6 In the definition of $\epsilon$ and in other places of this section, $\hat{\mathbf{p}}_{C_{2}}$ is treated as a dependent variable. It depends on $\hat{\mathbf{p}}_{C_{1}}$ in the following way

$$
\hat{\mathbf{p}}_{C_{2}}=R_{2}^{T} R_{1}\left(\hat{\mathbf{p}}_{C_{1}}+\mathbf{b}_{1}\right)-\mathbf{b}_{2} .
$$

In (51), the number $\epsilon$ is independent of the inertial frame $\{\mathcal{I}\}$.

REMARK 7 Since the LMIs derived in Theorems 5 and 6 are identical, Theorem 6 provides necessary and sufficient conditions for the existence of a positive definite solution to the LMI (54) as well. This, in turn, leads to the observation that the performance level $\gamma$ of the filter $\mathcal{F}_{3}$ is bounded below by $\sqrt{1 /\left(1-r_{x}\right)^{2} \epsilon}$, where $\epsilon$ is given in Theorem 8 .

REMARK 8 The filters used in this paper borrowed from the structure of the nonlinear observer proposed in [5]. Both filters are designed for a process model that exhibits linear dynamics and nonlinear measurement equations. In view of this fact, one is naturally driven to ask the following question: why not simply solve the measurement equation to obtain estimate of $\mathbf{p}_{c}$ that can in turn drive a linear filter with a much simpler structure? This technique was, in fact, applied in an erlier version of the work reported in [5]. However, as pointed out by the authors the latency inherent to this approach led to unacceptable results. This stemmed from the fact that the estimate of $\mathbf{p}_{c}$ obtained by the nonlinear solver from the measurement equation and used by the linear filter represented a "delayed version" of the true position.

Furthermore, the algorithm used by the nonlinear solver requires inverting the Jacobian. In a noisy environment this may lead to excessive noise amplification. This problem is entirely avoided by the filters proposed in this work as well as by the nonlinear observer in [5]. Finally, the gains used by every filter in this work are of the form similar to the gains of optimal filters obtained for the LTI case. This is important, since in the LTI case even if the output matrix is invertible the optimal gain does not require inversion of this matrix.

\section{EXAMPLE}

In this section we present design and simulation results for the filters $\mathcal{F}_{1}-\mathcal{F}_{3}$. The LMIs included in Theorems 4-8 were implemented in Matlab. The gains obtained by solving these LMIs were used to simulate response of a given filter to non-zero initial conditions and $\mathcal{L}_{2}$ measurement noise.

The simulation scenario involved a ship moving North at a constant speed of $10 \mathrm{~m} / \mathrm{s}$ and the aircraft performing left turn from the course of $-50(\mathrm{NE})$ with altitude decrease and glissade (Fig. 7). The initial

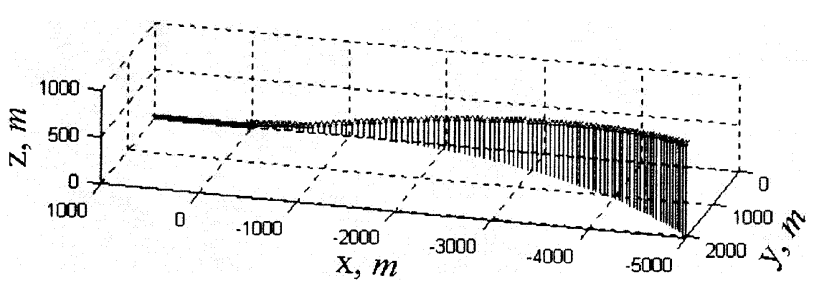

Fig. 7. 3D representation of simulation scenario.

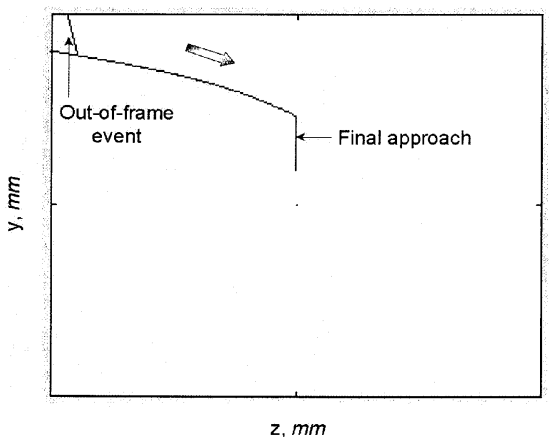

Fig. 8. Ship's smokestack projection in camera image plane during landing.

aircraft position is $[-5000 \mathrm{~m} ;-2000 \mathrm{~m} ;-1000 \mathrm{~m}]$ with respect to the ship's initial position, and its initial airspeed is $60 \mathrm{~m} / \mathrm{s}$. Initially, the aircraft is banking at an angle of $3.5 \mathrm{deg}$ to capture a 3 deg glideslope. Glideslope is characterized by reduced aircraft speed of $40 \mathrm{~m} / \mathrm{s}$ and begins approximately within $1100 \mathrm{~m}$ from the ship.

The onboard camera is fixed with -10 deg pitch angle with respect to aircraft's longitudinal axis. The projection of the ship's landing site onto the camera's image plane is shown in the Fig. 8. The jump in the upper left corner is due to a simulated out-of-frame event discussed later in this section.

Filters $\mathcal{F}_{1}-\mathcal{F}_{3}$ are employed sequentially as a function of range to the ship as shown in the Fig. 9. Fig. 10 summarizes the design parameters for each filter and shows time histories of the errors in position estimation for each filter in response to large initial errors and $L_{2}$ sensor noise. (In Fig. 9 the largest error corresponds to errors in $x$ component of the position vector, while the errors in $y$ and $z$ are the same).

Fig. 11 illustrates the influence of the size of the cube $\mathcal{P}_{c}$ and the parameter $d x$ on the value of $\epsilon$. It turns out, due to the simple geometry, that $\epsilon$ reaches a minimum value, at the corner $x_{\max }, z_{\min }$ of the cube $\mathcal{P}_{c}$ (see Fig. 12). This dependence can be approximated by the following expression

$$
\epsilon=\left(\frac{z_{\min }-d x}{x_{\max }^{2}}\right)^{2}
$$

which fits experimental data with mean-squared value of at least 0.9997. Fig. 11 also includes the graph of the best achievable value of $\epsilon$ obtained by solving the LMIs in Theorem 5. The intersection of the two lines 


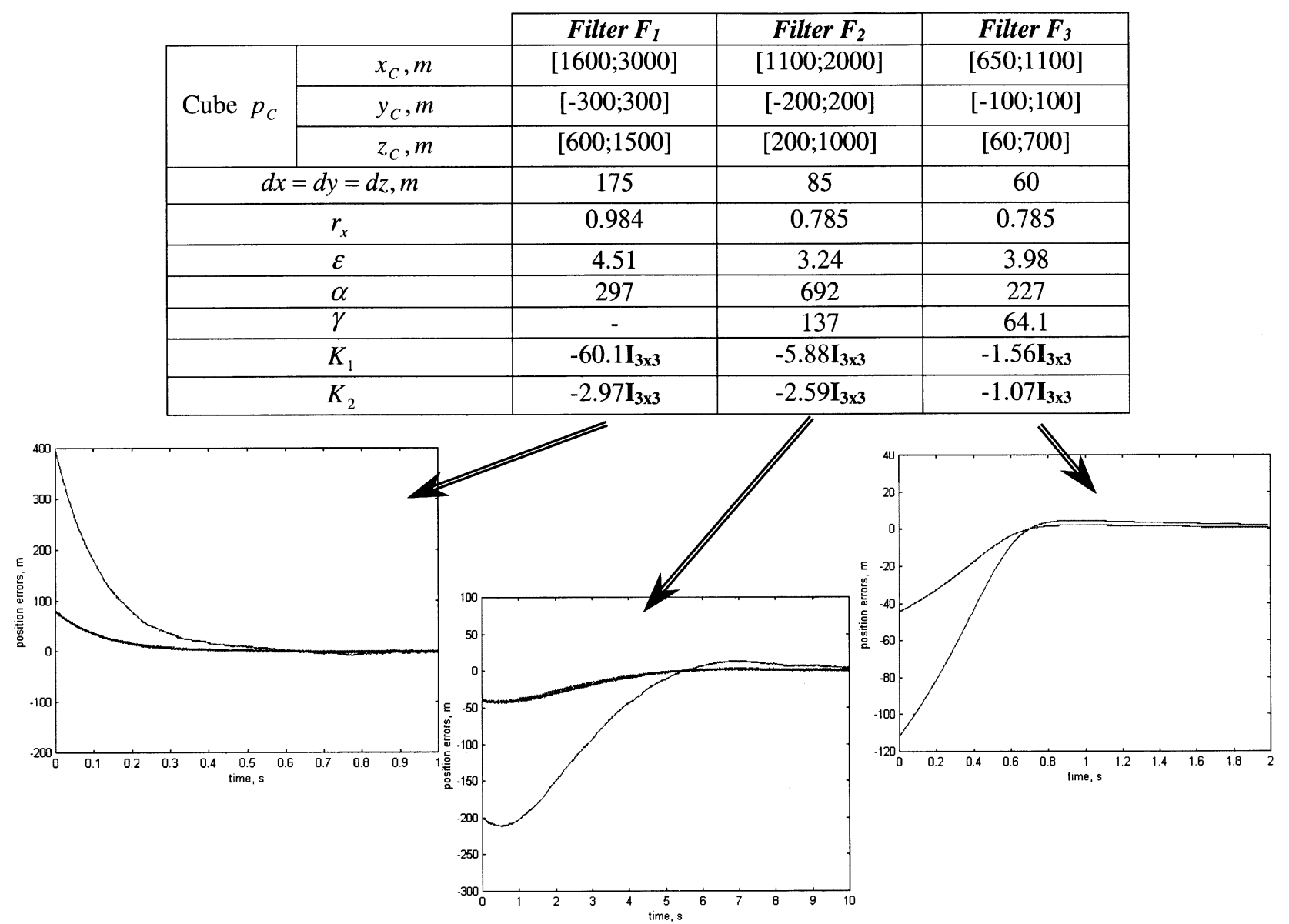

Fig. 9. Numerical results for filters $\mathcal{F}_{1}-\mathcal{F}_{3}$.

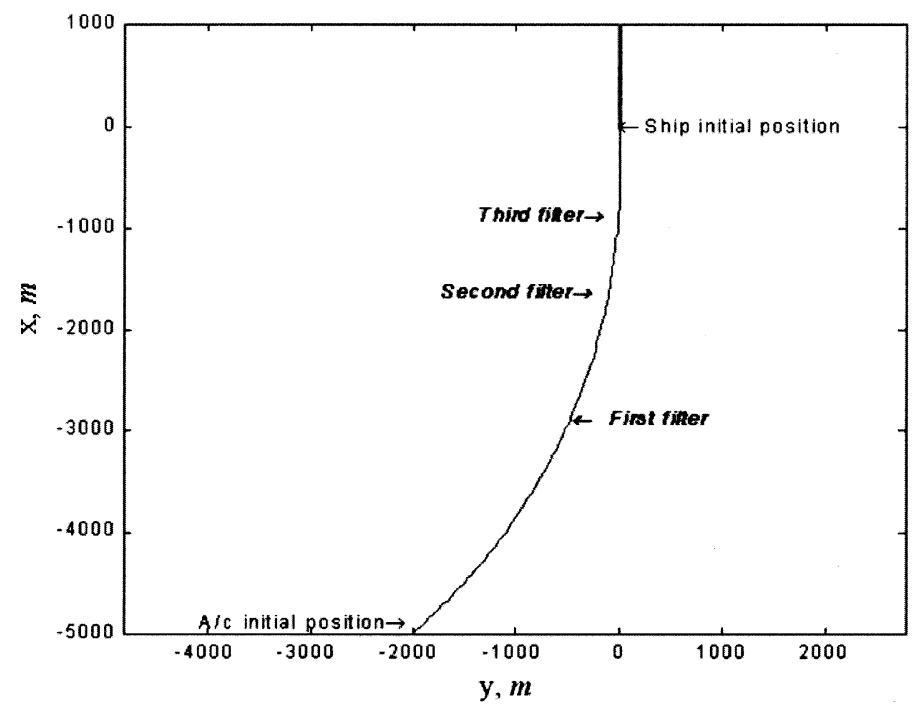

Fig. 10. Switching times between filters during landing.

provides the designer with size of the cube $\mathcal{P}_{c}$ where the filter $\mathcal{F}_{2}$ is guaranteed to have asymptotic stability and given $H_{\infty}$ performance characterized by $\gamma$.

The analytic expression (57) that shows dependence of $\epsilon$ on the size of the cube $\mathcal{P}_{c}$ can also be used to determine an estimate of the best achievable value of $\gamma$. As was shown in Section IV the lower bound $\gamma_{0}$ on the best achievable value of $\gamma$ is $\gamma_{0}=$ $1 /\left(1-r_{x}\right) \sqrt{\epsilon}$. This expression combined with (57) can be used to show dependence of $\gamma_{0}$ on the geometry of the problem as shown in Fig. 13.

Finally, Fig. 14 shows an example of recovery from an out-of frame event by the Filter $\mathcal{F}_{1}$. As can be seen in Fig. 8 (left top corner) after the elimination 


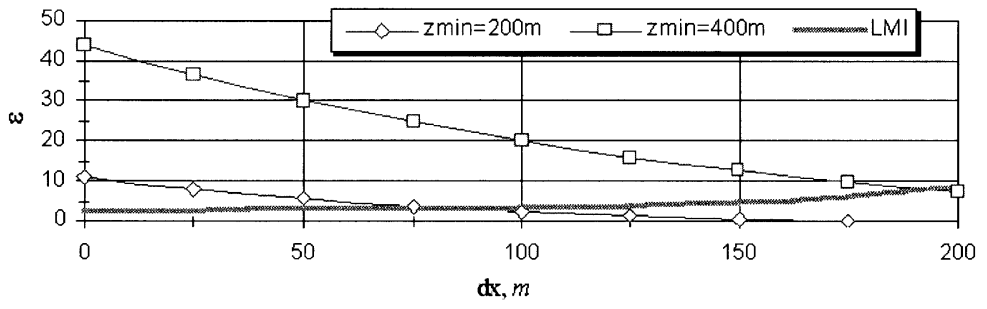

Fig. 11. Dependence of $\epsilon$ on value of $d x$ for filter $\mathcal{F}_{2}$.

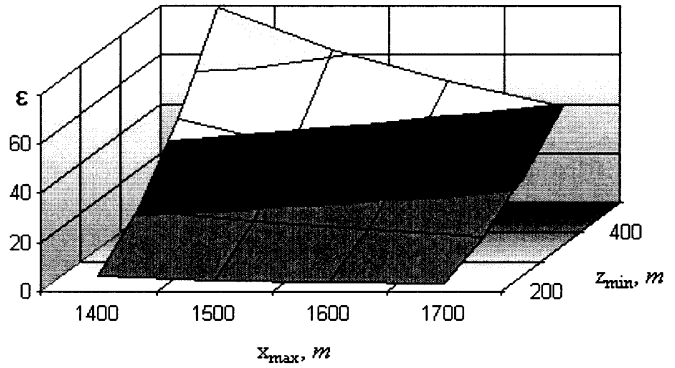

Fig. 12. Dependence of $\epsilon$ on values of $x_{\max }$ and $z_{\min }$ for filter $\mathcal{F}_{2}$.

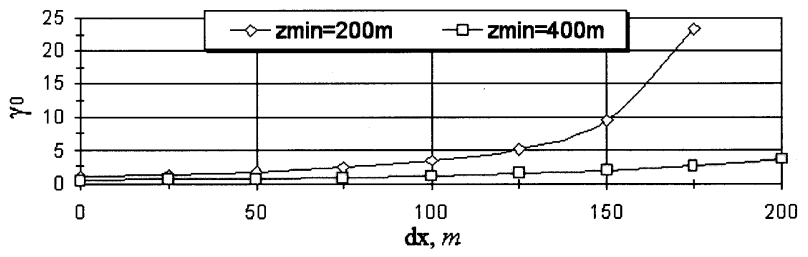

Fig. 13. Dependence of $\gamma$ on values on $d x$ for filter $\mathcal{F}_{2}$.VAD4

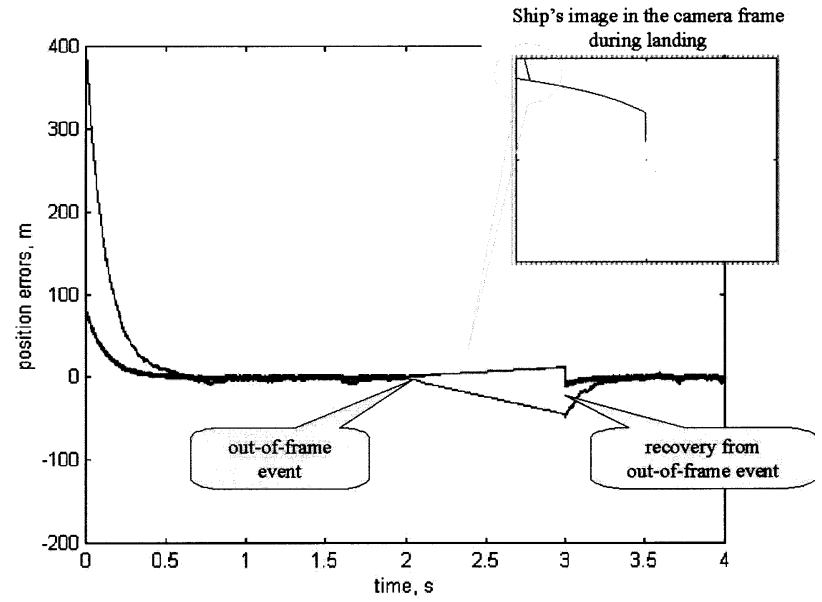

Fig. 14. Time history of $\mathcal{F}_{1}$ position errors during out-of-frame event.VAD4

of initial errors a disturbance in pitch causes an out-of frame event which lasts approximately $1 \mathrm{~s}$. At this point the feedback loops are turned off (see Fig. 15). Fig. 14 shows the resulting increase in the position error. As can be seen in Fig. 14 once the image is recaptured the accumulated errors are eliminated rapidly.

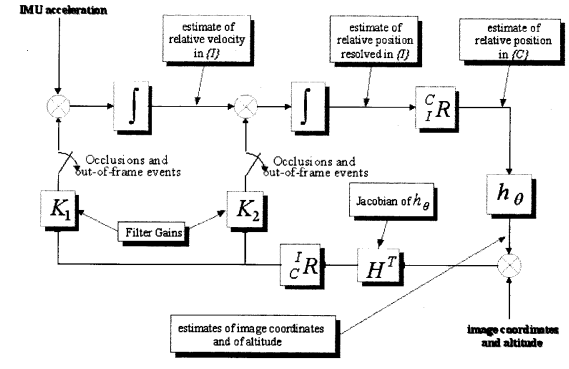

Fig. 15. Feedback loops in filters $\mathcal{F}_{1}$ and $\mathcal{F}_{2}$ turned off due to out-of-frame event or occlusion.

\section{CONCLUSIONS}

This paper addressed the problem of estimating the relative position and velocity of an aircraft with respect to a moving landing site such as a naval vessel. The problem was cast in the LPV framework and solved using tools that borrow from the theory of LMIs. This approach resulted in nonlinear filtering structures that integrate vision with inertial measurements and have regional stability and performance guarantees. Employing inertial sensors has an additional benefit of offering robustness with respect to out-of-camera events and occlusions, whereby these sensors can be used by the filter to provide the estimates of the image coordinates of the ship to the image processing algorithms. Furthermore, it was shown that the worst case $H_{\infty}$-based performance of these filters is bounded below by a quantity similar to the PDOP used in the science of navigation to determine the impact of geometry on the achievable performance of a navigation system.

Work in progress aims to implement the algorithms proposed in this paper. The critical hardware implementation issues include synchronizing the clocks on the vision and inertial sensors to properly match the image processing data and inertial data.

\section{REFERENCES}

[1] Bresle, Y., and Merhav, S. (1986)

On-line vehicle motion estimation from visual terrain information. Part II: Ground velocity and position estimation.

IEEE Transactions on Aerospace and Electronic Systems, AES-22, 5 (Sept. 1986), 588-604. 
[2] Bresle, Y., and Merhav, S. (1987)

Recursive image registration with application to motion estimation.

IEEE Transactions on Acoustic, Speech, and Signal

Processing, ASSP-35, 1 (Jan. 1987), 70-85.

[3] Chatterji, G., Menon, P., and Shridar, B. (1997)

GPS/machine vision navigation system for aircraft.

IEEE Transactions on Aerospace and Electronic Systems, 33, 3 (July 1997), 1012-1025.

[4] Chatterji, G., Menon, P., and Shridar, B. (1998)

Vision based position and attitude determination for aircraft night landing.

AIAA Journal of Guidance, Control and Dynamics, 21, 1 (Jan.-Feb. 1998), 84-92.

[5] Rizzi, A., and Koditscheck, D. (1996)

An active visual estimator for dexterous manipulation. IEEE Transactions on Robotics and Automation, 12, 5 (Oct. 1996), 697-713.

[6] Becker, G., and Packard, A. (1994)

Robust performance of linear, parametrically varying systems using parametrically-dependent linear, dynamic feedback.

Systems and Control Letters (1994).

[7] Boyd, S., El Ghaoui, L., Feron, E., and Balakrishnan, B.

(1994)

Linear Matrix Inequalities in Systems and Control Theory. Philadelphia: SIAM, Studies in Applied Mathematics, 1994.

[8] Green, M., and Limebeer, D. (1995)

Linear Robust Control.

Englewood Cliffs, NJ: Prentice-Hall, 1995.

[9] The MathWorks, Inc. (1997)

MatLab Application Toolbox, LMI Control.

The Math Works Inc., 1997.

[10] Scherer, C. (1997)

Generalized mixed $H_{2} / H_{\infty}$ synthesis for linear parameter varying systems.

Preprint, 1997.
[11] Britting, K. (1971)

Inertial Navigation System Analysis.

New York: Wiley-Interscience, 1971.

[12] Siouris, G. (1993)

Aerospace Avionics Systems: A Modern Synthesis.

New York: Academic Press Inc., 1993.

[13] Lentz, W. J. (1999)

NPS internal correspondence, 1999.

[14] Weng, J., Huang, T. S., and Ahuja, N. (1993)

Motion and Structure from Image Sequences.

New York: Springer-Verlag (Series in Information Sciences), 1993.

[15] Hager, G. (1997)

A modular system for robust positioning using feedback from stereo vision.

IEEE Transactions on Robotics and Automation, 13, 4

(Aug. 1997), 582-595.

[16] Nagpal, K., and Khargonekar, P. (1991)

Filtering and smoothing in an $H_{\infty}$ setting.

IEEE Transactions on Automatic Control, 36 (1991), 152-166.

[17] Brown, R. (1972)

Integrated navigation systems and Kalman filtering: A perspective.

Journal of the Institute of Navigation, 19, 4 (Winter 1972-1973), 355-362.

[18] Merhav, S. (1996)

Aerospace Sensor Systems and Applications.

New York: Springer-Verlag, 1996.

[19] Massatt, P., and Rundick, K. (1990)

Geometric formulas for dilution of precision calculations. Journal of Institute of Navigation, 37, 4 (Winter 1990-1991), 379-391.

[20] Parkinson and Spilker (Eds.) (1996)

Global Positioning System: Theory and Applications I. New York: AIAA, 1996.
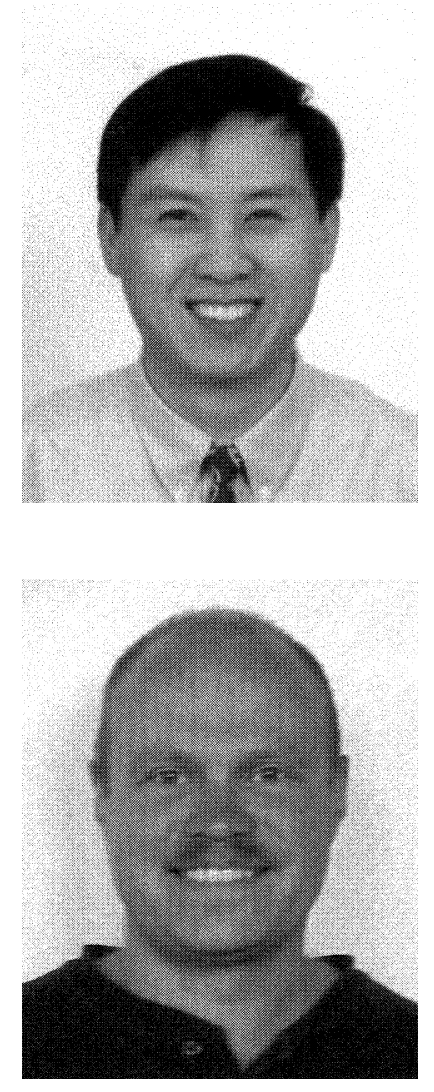

Wei Kang (S'91-M'91) received his B.S. degree in 1982 and his M.S. degree in 1985 from Nankai University, China, both in mathematics. In 1991, he received his Ph.D. degree in mathematics from the University of California at Davis.

From 1991 to 1994, he was a visiting assistant professor of systems science and mathematics at Washington University in St. Louis. He joined the faculty of the Naval Postgraduate School in 1994, where he is currently an associate professor of mathematics. His current research interest is nonlinear control theory and its applications. His published results include formation control, autonomous vehicle control, bifurcation control, normal forms and feedback invariants, nonlinear filtering, and $H$-infinity control.

Dr. Kang is a member of SIAM.

Oleg Yakimenko was born in Novosibirsk, Russia. He received the B.Sc. and M.Sc. degrees in computer science and control engineering from the Moscow Institute of Physics and Technology, Moscow, Russia in 1984 and 1986, respectively. In 1988 he received M.Sc. degree in aeronautical engineering and operations research from the Air Force Engineering Academy (AFEA). In the same academy he received the degree of the Candidate of Technical Sciences (Ph.D.) (1991) and Doctor of Technical Sciences (1996) specializing in optimal control theory and aeronautical engineering.

He has progressed through the professorial ranks at AFEA and is currently a visiting professor at the Naval Postgraduate School as a National Research Council Senior Research Associate. He is a specialist in the area of flight mechanics, optimal control, trajectory optimization, and human factors.

During his professional career he has supervised five M.Sc. and six Ph.D. students, written eleven textbooks and over one hundred articles. He is an Associate Fellow of the Russian Aviation and Aeronautics Academy of Sciences, and a Senior Member of AIAA. 